\title{
Software Radio Architecture with Smart Antennas: A Tutorial on Algorithms and Complexity
}

\author{
Javad Razavilar, Member, IEEE, Farrokh Rashid-Farrokhi, Member, IEEE, and K. J. Ray Liu, Senior Member, IEEE
}

\begin{abstract}
Recently, there has been considerable interest in using antenna arrays in wireless communication networks to increase the capacity and decrease the cochannel interference. Adaptive beamforming with smart antennas at the receiver increases the carrier-to-interference ratio (CIR) in a wireless link. This paper considers a wireless network with beamforming capabilities at the receiver which allows two or more transmitters to share the same channel to communicate with the base station. The concrete computational complexity and algorithm structure of a base station are considered in terms of a software radio system model, initially with an omnidirectional antenna. The software radio computational model is then expanded to characterize a network with smart antennas. The application of the software radio smart antenna is demonstrated through two examples. First, traffic improvement in a network with a smart antenna is considered, and the implementation of a hand-off algorithm in the software radio is presented. The blocking probabilities of the calls and total carried traffic in the system under different traffic policies are derived. The analytical and numerical results show that adaptive beamforming at the receiver reduces the probability of blocking and forced termination of the calls and increases the total carried traffic in the system. Then, a joint beamforming and power control algorithm is implemented in a software radio smart antenna in a CDMA network. This shows that, by using smart antennas, each user can transmit with much lower power, and therefore the system capacity increases significantly.
\end{abstract}

Index Terms-Adaptive beamforming, handoff, power control, smart antennas, software radio.

\section{INTRODUCTION}

$\mathbf{T}$ HE software radio is emerging from military applications. Because of recent advances in high-speed digital signal processors (DSP's) and analog-to-digital converters (ADC's), the commercial implementation of software radios has become viable. In software radio systems, the IF signal is digitized using wide-band ADC's, and all of the subsequent processing is implemented in software [1]-[8]. The main advantage of software radio is its great flexibility such that it can be pro-

Manuscript received November 15, 1997; revised April 2, 1998 and August 17, 1998.

J. Razavilar was with the Department of Electrical Engineering and the Institute for Systems Research, University of Maryland, College Park, MD 20742 USA. He is now with Corporate R\&D, 3COM Laboratories, San Diego, CA 92128 USA.

F. Rashid-Farrokhi was with the Department of Electrical Engineering and the Institute for Systems Research, University of Maryland, College Park, MD 20742 USA. He is now with the Wireless Communications Research Department, Bell Laboratories, Lucent Technologies, Holmdel, NJ 07733 USA.

K. J. R. Liu is with the Department of Electrical Engineering and the Institute for Systems Research, University of Maryland, College Park, MD 20742 USA.

Publisher Item Identifier S 0733-8716(99)02978-9. grammed for emerging standards. It also can be dynamically updated with new software without any changes in hardware and infrastructure. Rapid deployment is another important feature of the software radio. In wireless applications where different standards might be deployed, users' roaming can be a big issue in existing platforms. In the software radio system, users just need to download the new air interface upon entering the new territory.

The reallocation of bandwidth is a standards-setting process that takes a considerably long time. On the other hand, due to fixed finite resources, one cannot assume that more physical radio channels will be added to a spectrum allocation to reduce the probability of blocking or forced termination [9]-[13]. The increasing popularity of wireless communication services together with the limited amount of the available radio spectrum calls for highly efficient usage of resources (traffic channels) in the system [14]. The interference reduction capability of antenna arrays has been considered as a means to increase the capacity of wireless systems [15]-[21]. Specifically, using beamforming techniques at the receiver, two or more transmitters can share the same traffic channel to communicate with the base station at the same time. An adaptive antenna array is used at the base station to form several antenna beams simultaneously. Each beam captures one transmitter by automatically pointing its pattern toward that transmitter while nulling other cochannel transmitters and multipath signals [19]-[21]. In this way, the cochannel interference (CCI) is minimized, and therefore the carrier-to-interference ratio (CIR) for the signal of interest is maximized. In urban wireless environments, the signal transmitted by each user is reflected by surrounding buildings and the terrain. Therefore, several copies of the transmitted signal are received at the base station with different delays and different attenuation. This effect is called multipath fading. In space-only diversity, given a cell with $L$ channels, an $M$-element antenna array that forms $M-1$ distinct array nulls at the receiver allows a maximum of $K L$ users to be served in a cell, where $K=M / \alpha$ and $\alpha$ is the multipath factor representing the number of distinct strong path components received from each mobile station at the beamforming array. In space-time diversity, spatial nulls reject the CCI, while intersymbol interference (ISI) is eliminated by time diversity.

Sectorization is an alternative to smart antennas. Sectorized cell sites employ hardware beamforming array antennas in which each beam is assigned a distinct RF channel set. The direction and gain of sectorized antennas are fixed in the hardware. They cannot place nulls on interference like 
the smart antenna. The cost of a sectorized cell site is an increase in the (analog) hardware complexity of the cell site, but there is virtually no impact on the single-channel cell site software. When the capacity of a sectorized site is exceeded, one may then overlay a smart antenna with its dedicated digital signal processing (DSP) capacity and unique software architecture which accommodates the vastly increased computational demand of the beamforming network. The smart antenna's beams are not fixed, but are dynamic per user, and will place nulls that cancel interference. Another advantage of the smart antenna system is the reduced rate of handoffs because of tracking the user. In this paper, the term "cell" means either a conventional cell or one sector of a sectorized cell.

This paper first presents a tutorial review of beamforming algorithms. It then reviews a software radio architecture, first with omnidirectional antennas, and then with smart antennas. Two applications show the advantages of a system with a smart antenna. First, a wireless network with beamforming capabilities at the receiver allows two or more transmitters to share the same channel to communicate with the base station. Each cell is modeled by a multiuser multiserver service facility. Each server is a beamformed channel formed by the cell's base station. For a set of cochannel transmitters, the probability of successful capture by a separate antenna beam is computed. The success probabilities are taken into account in the queueing model of the system. From this generalized model, the closed-form blocking probabilities of the calls and total carried traffic in the system under different traffic policies are derived. The second application shows how to implement a robust power control algorithm when using a smart antenna at the base station. In CDMA networks, power control is an important issue because of the near-far effect. Therefore, the effective power control algorithm results in a capacity increase in the system.

The paper is organized as follows. In Section II, various adaptive beamforming algorithms are discussed. The software radio architecture in a system with an omnidirectional antenna and also with a smart antenna is presented in Section III. The complexity issue is also discussed in this section. Some applications and advantages of using software radio smart antennas are presented in Section IV, where traffic improvement and capacity enhancement are addressed. Section V includes our conclusions and remarks.

\section{REVIEW OF SPATIAL MUltipleXING USING ADAPTIVE BEAMFORMING}

A smart antenna is a multibeam adaptive array with its gain pattern adjusted dynamically [23]. For a fixed $M$-beam sectorized antenna and an $M$-element smart antenna system, the antenna gain and system capacity are increased approximately $M$-fold. In a fixed beam system, the handoff from one beam to another is the main disadvantage, while in a smart antenna system, each beam tracks its user within the coverage of the cell. Moreover, smart antenna systems provide diversity gain against multipath signals and strong interferers by placing antenna nulls toward those sources. Since the beamforming in

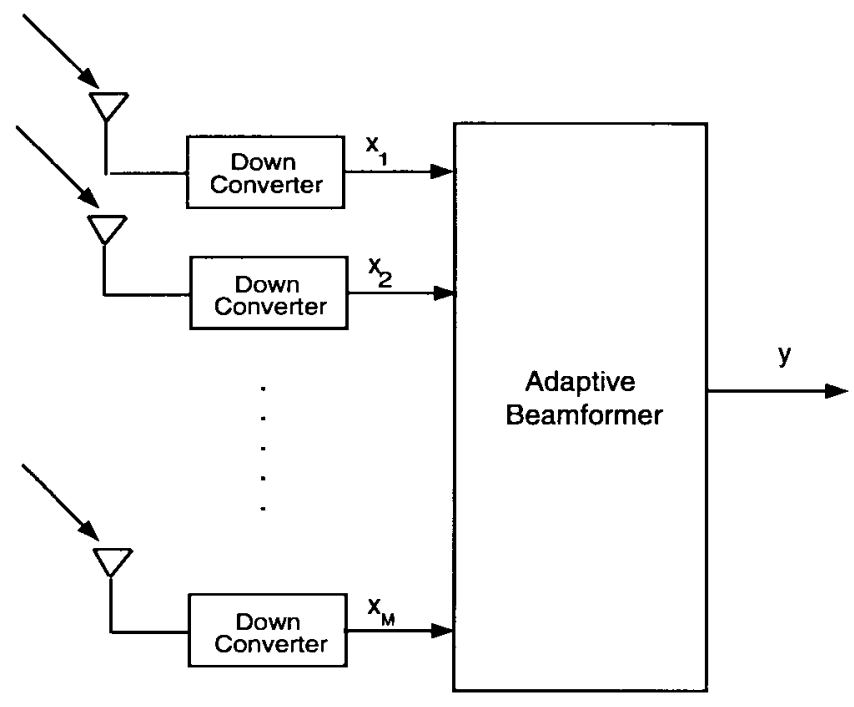

Fig. 1. A diversity combining system.

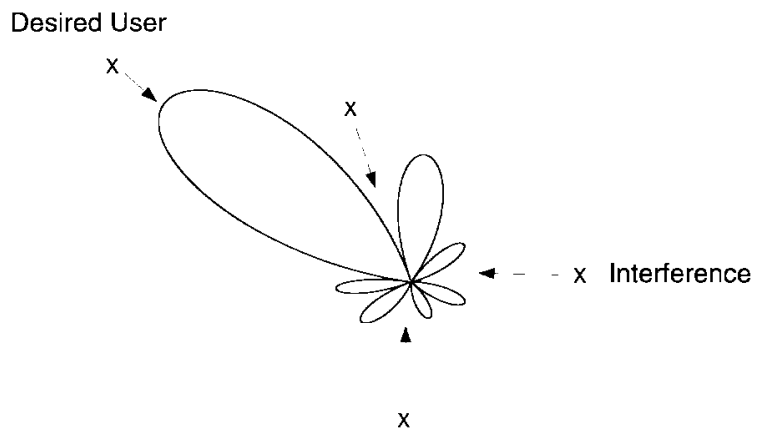

Fig. 2. Sample array pattern.

adaptive arrays is done in IF and baseband, these systems are well suited for software radio implementation.

Space and time diversity reduce the cochannel interference (CCI) and intersymbol interference (ISI). If the desired signal and the cochannel signals have different temporal or spatial signatures, time and space diversity can improve the signalto-noise ratio (SNR). The block diagram of an adaptive array is depicted in Fig. 1. In general, the objective is to form the main beam toward the desired user and nulls toward the interference sources as illustrated in Fig. 2.

An antenna array consisting of $M$ elements is considered at the receiver. Adaptive beamforming capabilities of antenna arrays may be used to maintain a constant gain for the signal along the direction of interest, and adjusting the nulls so as to reject the cochannel interference. In this way, the interference is minimized and the CIR for the signal of interest is maximized. A set of $J$ cochannel transmitters is considered. Let $s_{j}(k)$ for $j=1,2, \cdots, J$ denote the $j$ th transmitted signal. Assume that at most $N$ multipath signals from each user arrive in the base station with different delays $\left(\tau_{n}\right)$. Therefore, the received signal vector due to the $s_{i}(k)$ is

$$
\boldsymbol{x}(k)=\sum_{j=1}^{J} \sqrt{P_{j} G_{j}} \sum_{n=1}^{N} \alpha_{j}^{n} \boldsymbol{a}\left(\theta_{j}^{n}\right) s_{j}\left(t-\tau_{n}\right)+\boldsymbol{n}(k)
$$




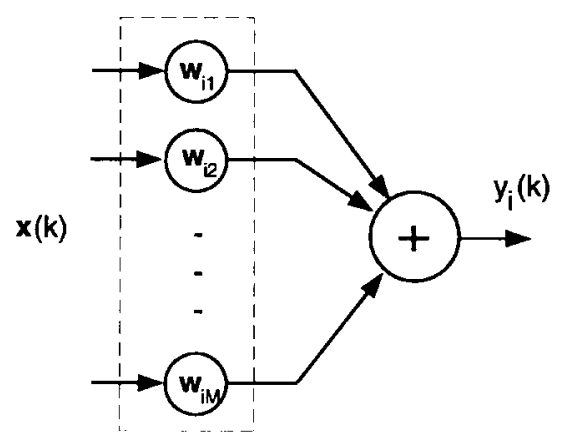

Fig. 3. Space diversity combiner.

where $\theta_{i}^{n}$ is the arrival direction of the $n$th multipath signal from the $i$ th user, $P_{i}$ is the power of the $i$ th transmitter, $G_{i}$ is the link gain between the $i$ th transmitter and the base station, $\alpha_{i}^{n}$ is the $n$th path fading coefficient, and $\boldsymbol{a}\left(\theta_{i}^{n}\right)$ is the array response to the multipath signal arriving from direction $\theta_{i}^{n}$ with

$$
\boldsymbol{a}\left(\theta_{i}^{n}\right)=\left[a_{1}\left(\theta_{i}^{n}\right), a_{2}\left(\theta_{i}^{n}\right), \cdots, a_{M}\left(\theta_{i}^{n}\right)\right]^{T} .
$$

\section{A. Space Diversity Combining}

In a space diversity system, the weighted sum of the received signal is combined at the output of beamformer as illustrated in Fig. 3. A weighted sum of the outputs of the array elements is generated by a beamformer in the following way:

$$
y_{i}(k)=\boldsymbol{w}_{i}^{H} \boldsymbol{x}(k)
$$

where $\boldsymbol{w}_{i}^{T}=\left[w_{i 1}, \cdots, w_{i M}\right]$ is the weight and $\boldsymbol{x}^{T}(k)=$ $\left[x_{1}(k), \cdots, x_{M}(k)\right]$ is the received signal vector sampled at the output of the downconverters.

Minimum Variance Distortionless Response (MVDR): One can choose the weight vector $w_{i}$ to steer a beam toward the direction of the signal of interest $\theta_{i}$ and adjust the nulls to reject the interference. This is done by attempting to maintain a distortionless response in the direction of interest and placing the nulls in the directions of other cochannel interferers. The average output power is given by

$$
E_{i}=E\left[y_{i}(k) y_{i}^{H}(k)\right]=w_{i}^{H} E\left[\boldsymbol{x}(k) \boldsymbol{x}^{H}(k)\right] \boldsymbol{w}_{i}=\boldsymbol{w}_{i}^{H} \boldsymbol{\Phi}_{i} w_{i}
$$

where

$$
\begin{aligned}
\boldsymbol{\Phi}_{i}= & P_{i} G_{i} \alpha_{i}^{1} \boldsymbol{a}_{i}\left(\theta_{i}^{1}\right) \boldsymbol{a}_{i}^{H}\left(\theta_{i}^{1}\right) \\
& +\sum_{j=1, j \neq i}^{J} P_{j} G_{j} \sum_{n=2}^{N} \alpha_{j}^{n} \boldsymbol{a}_{j}\left(\theta_{j}^{n}\right) \boldsymbol{a}_{j}^{H}\left(\theta_{j}^{n}\right)+\frac{N_{0}}{2} I .
\end{aligned}
$$

The correlation matrix due to the interference terms is

$$
\boldsymbol{\Phi}_{N}=\sum_{j=1, j \neq i}^{J} P_{j} G_{j} \sum_{n=2}^{N} \alpha_{j}^{n} \boldsymbol{a}_{j}\left(\theta_{j}^{n}\right) \boldsymbol{a}_{j}^{H}\left(\theta_{j}^{n}\right)+\frac{N_{0}}{2} I .
$$

If the array response in the direction of the desired user is known, the beamformer tries to minimize the output power $E_{i}$ subject to maintaining a distortionless response in the direction of interest such that $w_{i}^{H} \boldsymbol{a}_{i}\left(\theta_{i}^{1}\right)=1$. For this reason, this adaptive beamformer is called an MVDR [15] beamformer. From (4) and (5), with the constraint that $\boldsymbol{w}_{i}^{H} \boldsymbol{a}_{i}\left(\theta_{i}^{1}\right)=1$, the received signal power plus the interference power as a function of $w_{i}$ is

$$
\begin{aligned}
E_{i}= & P_{i} G_{i}+\sum_{j=1, j \neq i}^{J} P_{j} G_{j} \sum_{n=2}^{N} \alpha_{j}^{n} \boldsymbol{w}_{i}^{H} \boldsymbol{a}_{j}\left(\theta_{j}^{n}\right) \boldsymbol{a}_{j}^{H}\left(\theta_{j}^{n}\right) \boldsymbol{w}_{i} \\
& +\frac{N_{0}}{2} \boldsymbol{w}_{i}^{H} \boldsymbol{w}_{i} .
\end{aligned}
$$

Let $I_{i}$ denote the total interference plus noise power given by

$I_{i}=\sum_{j=1, j \neq i}^{J} P_{j} G_{j} \sum_{n=2}^{N} \alpha_{j}^{n} \boldsymbol{w}_{i}^{H} \boldsymbol{a}_{j}\left(\theta_{j}^{n}\right) \boldsymbol{a}_{j}^{H}\left(\theta_{j}^{n}\right) \boldsymbol{w}_{i}+\frac{N_{0}}{2} \boldsymbol{w}_{i}^{H} \boldsymbol{w}_{i}$.

In (7), $P_{i} G_{i}$ is the received power from the signal of interest, while $I_{i}$ given by (8) is the contribution to the output power $E_{i}$ from the interference and noise.

The optimum weight vector minimizes the interference $I_{i}$ while maintaining a unity gain in the direction of interest by imposing $\boldsymbol{w}_{i}^{H} a_{i}\left(\theta_{i}^{1}\right)=1$. The solution of this problem is

$$
\hat{\boldsymbol{w}}_{i}=\frac{\Phi^{-1} \boldsymbol{a}_{i}\left(\theta_{i}^{1}\right)}{\boldsymbol{a}_{i}^{H}\left(\theta_{i}^{1}\right) \Phi^{-1} \boldsymbol{a}_{i}\left(\theta_{i}^{1}\right)} .
$$

Since the desired signal (arriving along $\theta_{i}$ ) will not be affected by the beamforming process and only the interference is rejected, the CIR is maximized for the signal in the direction of interest, i.e.,

$$
\frac{\Gamma_{i, \max }=}{\sum_{j=1, j \neq i}^{J} P_{j} G_{j} \sum_{n=2}^{N} \alpha_{j}^{n} \hat{\boldsymbol{w}}_{i}^{H} \boldsymbol{a}_{j}\left(\theta_{j}^{n}\right) \boldsymbol{a}_{j}^{H}\left(\theta_{j}^{n}\right) \hat{\boldsymbol{w}}_{i}+\frac{N_{0}}{2} \hat{\boldsymbol{w}}_{i}^{H} \hat{\boldsymbol{w}}_{i}} .
$$

Minimum Mean-Square Error (MMSE): If the array response is not known, one may employ a training sequence and minimize the difference between the training sequence and the output of the beamformer in the mean-square sense

$$
\hat{\boldsymbol{w}}_{i}=\arg \min _{w_{i}} E\left[\left|d_{i}-\boldsymbol{w}_{i}^{H} \boldsymbol{a}_{i}\left(\theta_{i}^{1}\right)\right|^{2}\right] .
$$

The solution to this problem is given by [14]

$$
\hat{\boldsymbol{w}}_{i}=\Phi_{i}^{-1} p_{i}
$$

where $\Phi_{i}$ is defined as before and $\boldsymbol{p}_{i}$ is the cross correlation between the received vector and the training sequence; $\boldsymbol{p}_{\boldsymbol{i}}=$ $E\left[\boldsymbol{x}_{i} d_{i}^{*}\right]$. The maximum CIR in this case is given by

$$
\frac{\Gamma_{i, \max }=}{\left.\sum_{j=1, j \neq i}^{J} P_{j} G_{j} \sum_{n=2}^{N} \alpha_{i} \hat{\boldsymbol{w}}_{i}^{H} \hat{\boldsymbol{w}}_{i}\left(\theta_{i}^{H}\right) \boldsymbol{a}_{i}^{H}\left(\theta_{i}^{1}\right) \boldsymbol{w}_{i}\right) \boldsymbol{a}_{j}^{H}\left(\theta_{j}^{n}\right) \hat{\boldsymbol{w}}_{i}+\frac{N_{0}}{2} \hat{\boldsymbol{w}}_{i}^{H} \hat{\boldsymbol{w}}_{i}} .
$$


$\mathbf{X}(\mathrm{k})$

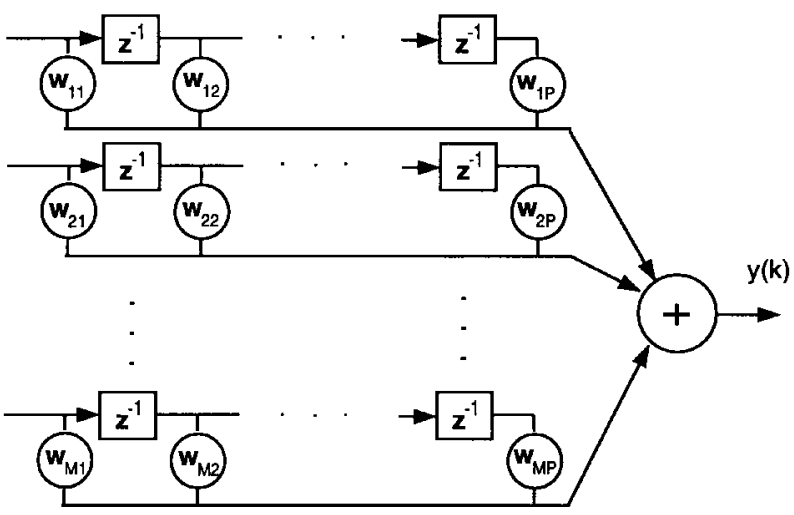

Fig. 4. Space-time diversity combiner.

\section{B. Space-Time Diversity Combining}

In the space diversity combining system of Fig. 3, the interference and multipath signals are rejected by placing nulls at the directions of those signals. In a broadband linear combiner, illustrated in Fig. 4, the desired signal and its multipath are combined at the combiner output to estimate the desired signal.

MMSE Space-Time Combining: The output of a broadband combiner can be expressed as

$$
\boldsymbol{y}(k)=\sum_{m=1}^{M} \boldsymbol{w}^{H}(m) \boldsymbol{x}(m)
$$

where $\boldsymbol{w}^{T}(m)=\left[w_{m 1}, \cdots, w_{m P}\right]$ is the weighting vector and $\boldsymbol{x}^{T}(m)=\left[x_{m 1}, \cdots, x_{m P}\right]$ is the received signal vector at the $m$ th element equalizer.

Let $\boldsymbol{W}=\left[\boldsymbol{w}^{T}(1), \cdots, \boldsymbol{w}^{T}(P)\right]^{T} \quad$ and $\boldsymbol{X}=$ $\left[\boldsymbol{x}(1)^{T}, \cdots, \boldsymbol{x}^{T}(P)\right]^{T}$. The output of the beamformer can be written as

$$
\boldsymbol{y}(k)=W^{H} X .
$$

In this case also, the objective is to minimize the mean-square error between the output of combiner and the desired signal $d$, i.e.,

$$
\hat{W}=\arg \min _{\boldsymbol{W}}\left|d-W^{H} \boldsymbol{X}\right|^{2} .
$$

The optimal beamformer coefficients are similar to space diversity MMSE by

$$
\hat{W}=E\left\{\boldsymbol{X} \boldsymbol{X}^{H}\right\}^{-1} E\left\{\boldsymbol{X} \boldsymbol{d}^{*}\right\} .
$$

Many adaptive methods that update weight vectors according to the incoming data have been developed [51], such as recursive least square (RLS) [21] and minimum mean-square error (MMSE) [23].

Blind Methods: Using a training sequence consumes some bandwidth. In order to save the bandwidth allocated to the training sequence, one may use blind methods. For singleinput single-output (SISO) systems, numerous blind identification algorithms [24]-[27] and blind equalization algorithms [28]-[37] have been proposed that exploit the higher order statistics of channel output. Among these algorithms, the Godard algorithm (GA) [30], also known as the constant

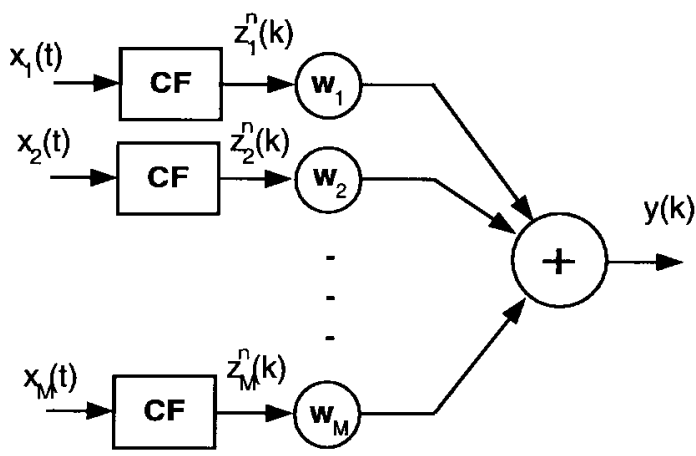

Fig. 5. Space-time diversity combiner in a CDMA system.

modulus algorithm (CMA) [35], [36], is one of the best and simplest adaptive blind equalization algorithms. Singleinput multiple-output (SIMO) systems may be viewed as fractionally spaced sampled communication systems which receive the distorted versions of one input signal. Fractionally spaced CMA adaptive blind equalizers under symbol timing offsets is considered in [38]. The equalization of multipleinput multiple-output (MIMO) transmission systems is studied in [39] and [40], where the MIMO channel impulse response is known. When the channel parameters of the MIMO systems are unknown, blind identification and equalization techniques must be used to separate and capture signals. The capture properties of the CMA algorithm used in MIMO systems with constant modulus input signals are studied in [36], [41], and [42]. The blind identification of MIMO systems using secondorder statistics or higher order statistics are also studied in [43]-[46]. Some subspace algorithms with fast convergence rate are proposed in [47].

\section{Space-Time Diversity in CDMA Systems}

In code-division multiple-access systems (CDMA), all users are sharing the same channel and each user has a different pseudonoise (PN) sequence. The received signal due to $J$ cochannel users at the receiver is given by

$$
\boldsymbol{x}(k)=\sum_{j=1}^{J} P_{j} G_{j} \sum_{n_{b}} s_{n_{b}} p_{j}\left(t-n_{b} T\right)+\boldsymbol{n}(k)
$$

where $\boldsymbol{x}(k)$ is the received signal at the array, $s_{n_{b}}$ is the transmitted sequence, and

$$
p_{j}(k)=\sum_{n} \boldsymbol{a}_{j}\left(\theta_{n}\right) \alpha_{j}^{n} c_{j}\left(t-\tau_{j}^{n}\right) .
$$

$\tau_{j}^{n}$ is the $n$th path delay associated with the $j$ th user, and $c_{j}$ is the $j$ th user PN sequence. In a space-diversity combiner, the signals from the main path are despread and combined at the beamformer output, as shown in Fig. 5. Code filters (CF) are matched to the desired user code and the delay is matched to the $p$ th path delay, i.e.,

$$
z_{j}^{n}(k)=\int_{T} \boldsymbol{x}(k) c_{j}\left(t-k T-\tau_{j}^{n}\right) d t
$$

and

$$
y_{j}^{n}(k)=\boldsymbol{w}^{H} z_{j}^{n}(k)
$$




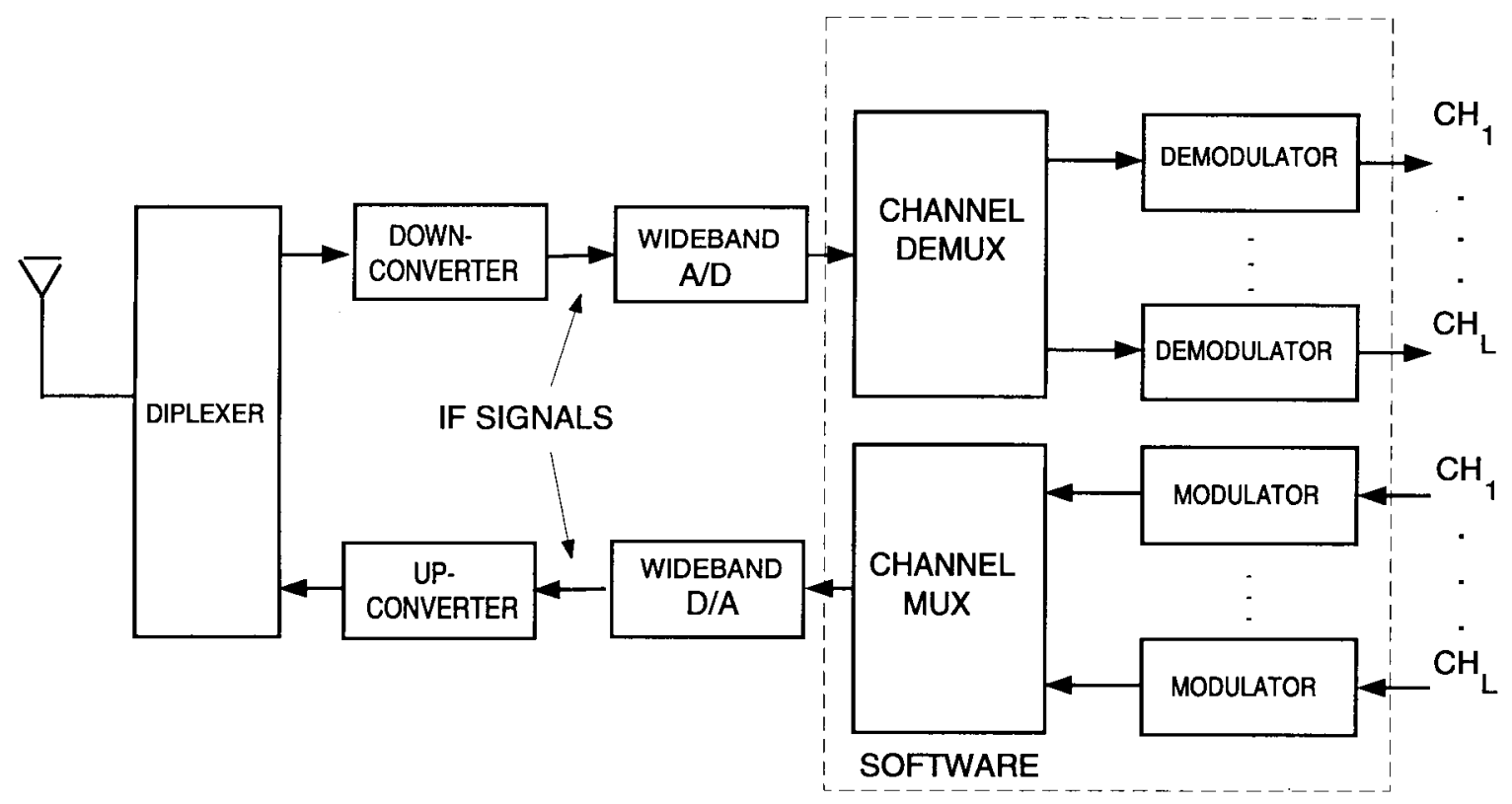

Fig. 6. Functional block diagram of the software radio for a base station with single omnidirectional antenna.

It has been shown [16] that the optimal beamformer for the above architecture is given by

$$
\hat{\boldsymbol{w}}=\frac{\Phi_{i n}^{-1} \boldsymbol{a}\left(\theta_{j}^{n}\right)}{\boldsymbol{a}\left(\theta_{j}^{n}\right)^{H} \Phi_{i n}^{-1} \boldsymbol{a}\left(\theta_{j}^{n}\right)}
$$

where $\Phi$ and $\Phi_{i n}$ are defined as before, and

$$
\Phi_{\text {in }}=\frac{\rho}{\rho-1}\left(\Phi-\frac{1}{\rho} \Phi_{o}\right)
$$

where $\rho$ is the processing gain and

$$
\Phi_{o}=\Phi_{i n}+\operatorname{Pa}\left(\theta_{j}^{n}\right) \boldsymbol{a}\left(\theta_{j}^{n}\right)^{H} .
$$

If the delay spread is larger than a chip period, a correlator may be employed for the signal received from each path. In signal antenna systems, signals from different correlators may be combined using maximum ratio combining. In those receivers, known as Rake receivers, each correlator is called a finger of the Rake receiver. In a multiple-antenna system, one can design a beamformer for each resolvable path, and then combine the outputs with a standard Rake receiver [48], i.e.,

$$
\hat{z}_{j}(k)=\sum_{n=1}^{N} z_{j}^{n}(k) \alpha_{j}^{n}
$$

where $N$ is the maximum number of paths in each link.

\section{Software Radio Architecture with Smart Antenna}

The software radio allows one to build flexible, multiband radio systems rapidly. The functional block diagram of the software radio in a system with an omnidirectional antenna is shown in Fig. 6. The architecture of the software radio is described in more detail in [1]. As illustrated in this figure, a wide-band front end downconverts the received signal to the IF where it is sampled and digitized by a high-speed
ADC. The rest of the processing is implemented in software [2]. Such a radio may be dynamically updated with new software without changes in hardware and infrastructure [2]. One may achieve better performance by introducing coding on the bitstream using coding gain to improve the bit-error rate (BER). In the case of the omnidirectional antenna, the software of the cell site may either have an IF software radio architecture, in which the waveforms are synthesized and received in software, or they may have a baseband DSP radio architecture, in which the software is limited to bitstream signal processing. There are $P$ MIPS of processing capacity required per server, with $\mathrm{P}(\mathrm{IF}+$ baseband $) \gg P$ (baseband). A block diagram of a typical digital downconverter is depicted in Fig. 7. The wide-band IF signal is translated to a complex baseband signal by the quadrature multiplier. The numerically controlled oscillator (NCO) block generates the quadrature signals for the multipliers. Then the complex baseband signal is low-pass filtered to prevent aliasing due to decimation [5]. In a software radio architecture, these blocks are implemented in software. The high sampling rate of a wide-band receiver allows time for only a few operations per sample; however, the digital downconverter combines the frequency translation and filtering in one step. The specific signal processing steps show how the multiplication and convolution required to perform the frequency shift and filtering may be combined. The first step is frequency translation of the real-valued received signal samples $r[n]$ to baseband by multiplication with the appropriate complex exponential as follows:

$$
\begin{aligned}
y[n] & =r[n] e^{-j 2 \pi f_{c} n T_{s}} \\
& =r[n]\left\{\cos \left(2 \pi f_{c} n T_{s}\right)-j \sin \left(2 \pi f_{c} n T_{s}\right)\right\}
\end{aligned}
$$

where $f_{c}$ is the carrier frequency before translation to baseband and $T_{s}$ is the sample interval. The next step is to filter $y[n]$ 


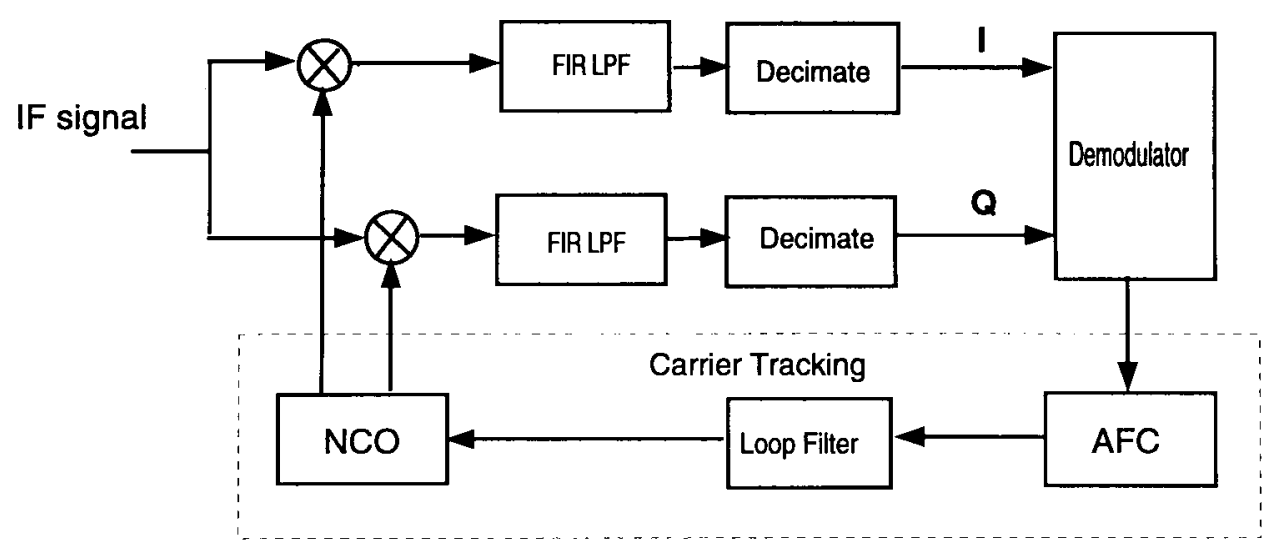

Fig. 7. Block diagram of the down converter and demodulator.

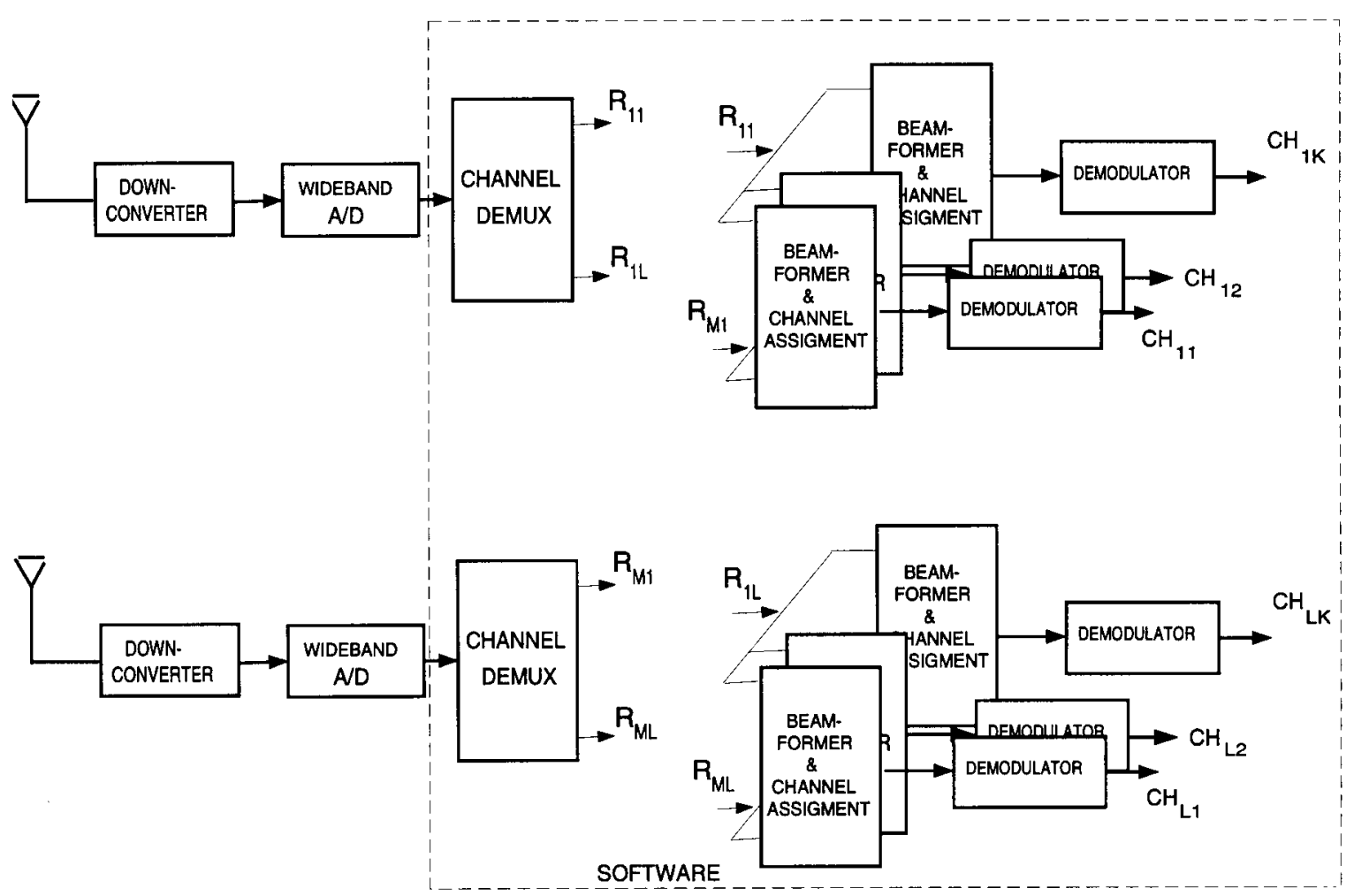

Fig. 8. Functional block diagram of the software radio for a base station with smart antenna.

with an FIR filter with filter coefficients $h[n]$

$$
\begin{aligned}
x[n] & =\sum_{m=0}^{M} h[m] y[n-m] \\
& =\sum_{m=0}^{M} h[m] r[n-m] e^{-j 2 \pi f_{c}(n-m) T_{s}} .
\end{aligned}
$$

From (14) and (15), combine these two processes as follows:

$$
\begin{aligned}
x[n] & =e^{-j 2 \pi f_{c} n T_{s}} \sum_{m=0}^{M} h[m] y[n-m] \\
& =\sum_{m=0}^{M} h[m] r\left[n-m e^{-j 2 \pi f_{c} m T_{s}}\right. \\
& =e^{-j 2 \pi f_{c} n T_{s}} \sum_{m=0}^{M} c[m] r[n-m]
\end{aligned}
$$

where $c[m]=h[m] e^{j 2 \pi f_{c} n T_{s}}$. Here, not only is the number of computations reduced, but there is no need to compute the unfiltered baseband signal $y[n]$, which further reduces the number of operations [5].

The functional block diagram of the software radio with smart antennas is shown in Fig. 8. Each antenna element has its own downconverter and ADC. But the subsequent beamforming and demodulation are implemented in software and are shared among all of the elements. Fig. 9 illustrates the software architecture of the beamformer for each channel. The received signal from each antenna element is passed through the same software block as depicted in Fig. 7 to generate $I$ and $Q$ signals. These signals are combined using the "combiner" block as shown in Fig. 9. The received signal from the user would be the output of the demodulator in Fig. 9. Using $K$ similar blocks for each channel one allows up to $K$ users to share the same channel. Since each user generates its carrier 


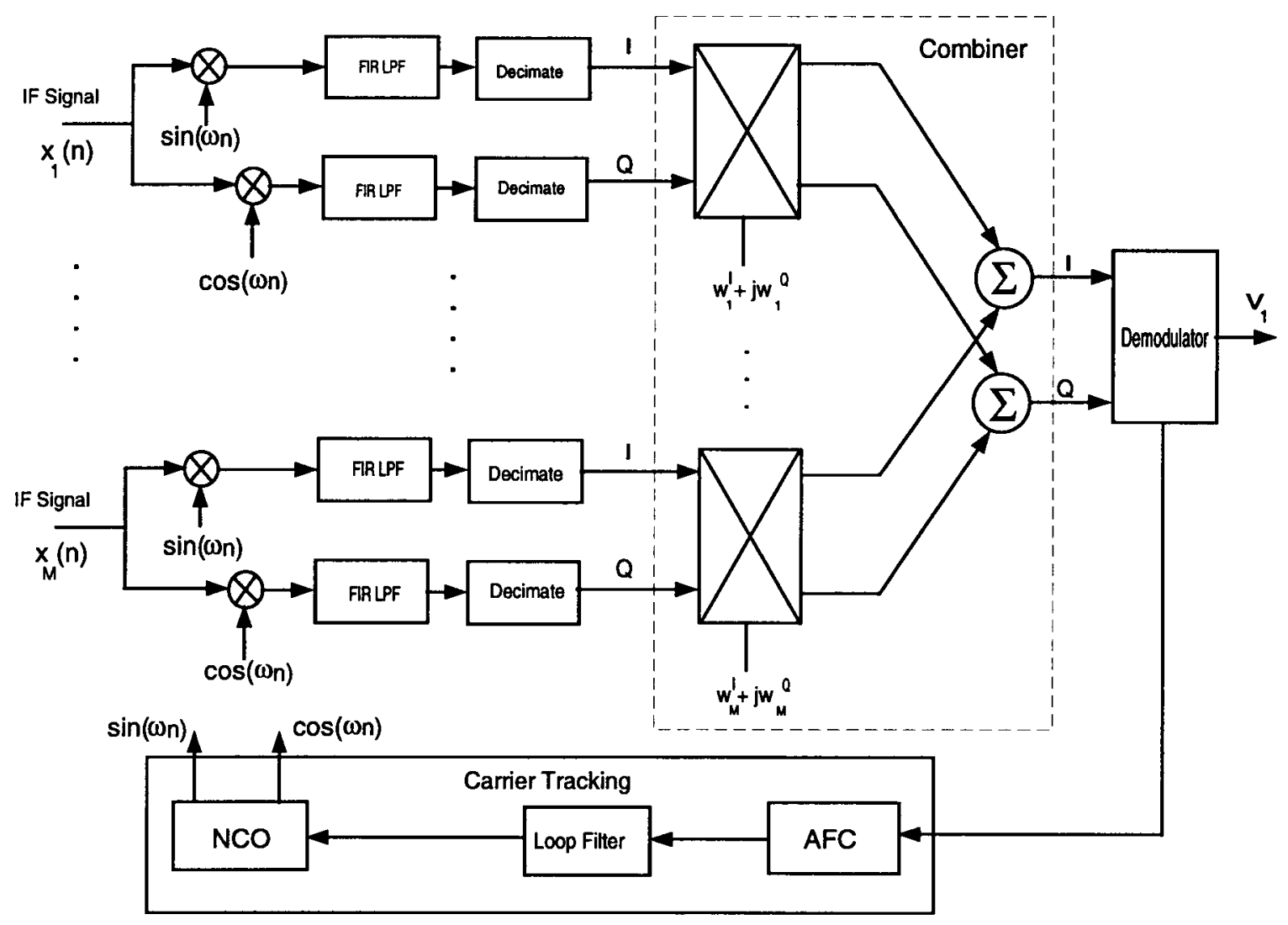

Fig. 9. Block diagram of the software beamformer for each user.

TABLE I

Computational Complexity of Various Beamforming Algorithms

\begin{tabular}{l|l|l|l}
\hline Algorithm & Multiplications & Divisions & Additions \\
\hline$L M S$ & $2 Q+1$ & 0 & $2 Q$ \\
\hline$R L S$ & $2 Q^{2}+7 Q+5$ & $Q^{2}+4 Q+3$ & $2 Q^{2}+6 Q+4$ \\
\hline$F T F$ & $7 Q+12$ & 4 & $6 Q+3$ \\
\hline$L S L$ & $10 Q+3$ & $6 Q+2$ & $8 Q+2$ \\
\hline
\end{tabular}

locally, there are separate carrier tracking and downconverting blocks per user. It was mentioned that one can assign $K$ users to each channel by using $K$ beams for each channel. In this manner, a system with $L$ physical channels serves at most $K L$ users. Such a software radio with a smart antenna is scalable. Increasing the number of beams and users is a software process within the constraints of the hardware costs. The proportion of increase in computational resources required for the smart antenna depends on the cell site architecture. With baseband DSP, one converts the smart antenna channels back to analog IF to provide only $L$ channels in the conventional cell site as the maximum capacity of each cell. In principle, with an IF software radio architecture, the smart antenna processing could be part of the larger pooled DSP resources, and one could implement $K L$ or $K L / \alpha$ service channels. In the sequel, a software radio architecture is assumed in which one synthesizes up to $K L$ servers in the integrated DSP architecture of the software radio cell site. As illustrated in Fig. 8, the beamforming process can be implemented on DSP's. Usually, adaptive algorithms are used to update the weight vectors. The

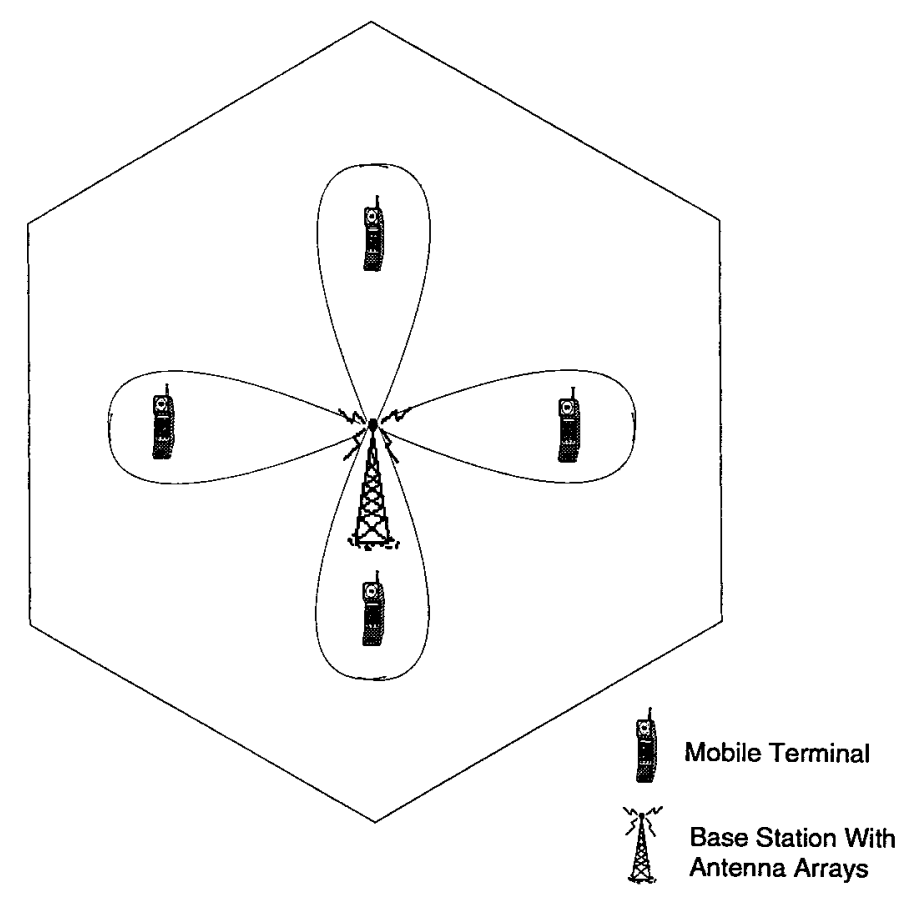

Fig. 10. Capturing four mobile users over the same channel successfully by an adaptive antenna array at the BS.

optimum beamformer weight vector presented in the previous section requires knowledge of second-order statistics. These statistics are usually not known, but with the assumption of ergodicity, they can be estimated. Statistics may also change 


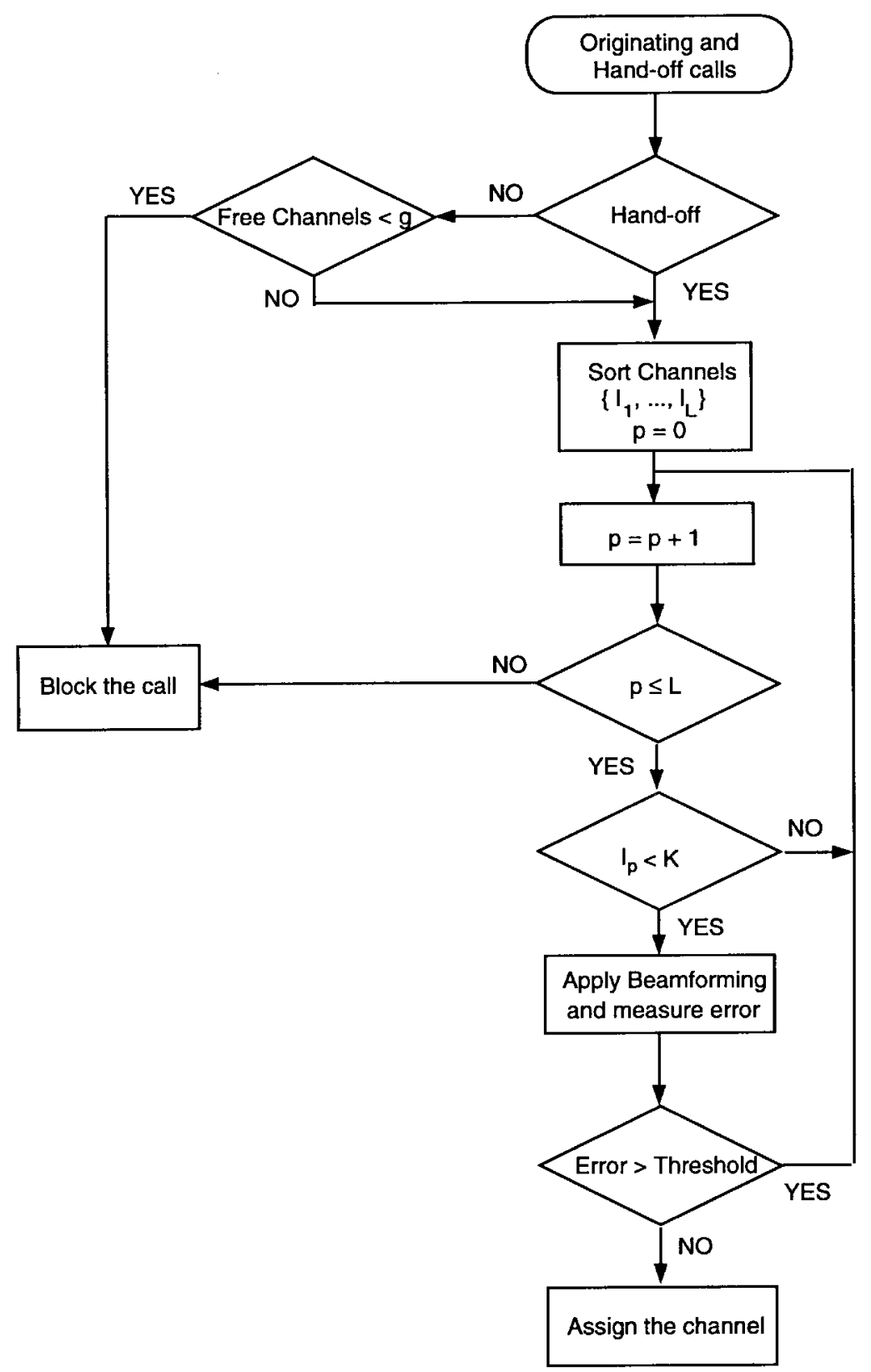

Fig. 11. Flow chart of the channel assignment algorithm in a system with smart antenna.

over time (due to moving interferers). To solve these problems, weight vectors are typically determined adaptively. One may employ training sequences to update the weight vectors. The training sequence is known at the receiver. Let $d_{i}, i=1, \cdots, Q$ denote the training sequence. The received training sequence at the receiver is denoted by $X_{i}, i=1, \cdots, Q$.

The direct matrix inversion (DMI) is the straightforward method for calculating the weight vectors. DMI minimizes the difference between the training sequence and the output of the beamformer in a mean-square sense

$$
\hat{\boldsymbol{w}}_{i}=\arg \min _{w_{i}} \sum_{q=1}^{Q}\left|d_{i}-\boldsymbol{w}_{i}^{H} \boldsymbol{X}_{i}(q)\right|^{2} .
$$

The solution to this problem is given by [14]

$$
\hat{w}_{i}=\Phi_{i}^{-1} \boldsymbol{p}_{i}
$$

where $\Phi_{i}$ is the estimated correlation matrix and $\boldsymbol{p}_{i}$ is the cross correlation between the received vector and the training sequence given by

$$
\begin{aligned}
\Phi_{i} & =\frac{1}{Q} \sum_{q=1}^{Q} \boldsymbol{X}_{i}(q) \boldsymbol{X}_{i}^{H}(q) \\
\boldsymbol{p}_{i} & =\frac{1}{Q} \sum_{q=1}^{Q} \boldsymbol{X}_{i}(q) \boldsymbol{d}_{i} .
\end{aligned}
$$

The complexity of the DMI method is $O\left(Q^{3}\right)$ where $Q$ is the length of the training sequence. Adaptive algorithms that update the weight vector taps reduce the complexity. In recursive estimation procedure the weights are adjusted iteratively. Such an approach eliminates the estimation of the correlation matrix or cross-correlation vector frequently as in 


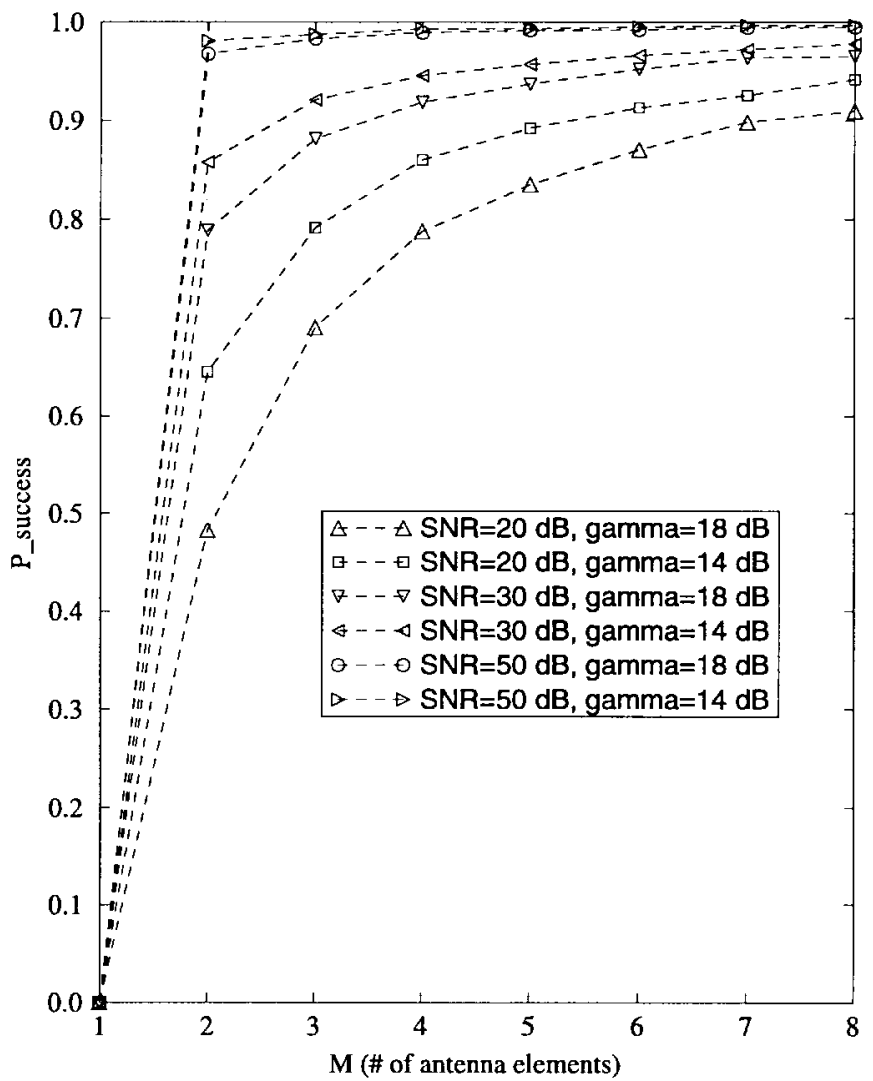

Fig. 12. Success probability $p_{2 \mid 1, M}$ for a 2-beam adaptive array for different values of $M$ (\# of antenna elements), SNR and threshold $\gamma$.

the DMI method. Moreover, each iteration trains the weight vector taps to track the incoming signal more accurately. Finally, at the end of the training sequence (with length $Q$ ), the new weight vector $w$ emerges after the $Q$ th iteration satisfying (17). The complexity of the least mean-square (LMS) algorithm, least square lattice (LSL) algorithm, fast transversal filter (FTF) algorithm, and QRD-RLS algorithm increases linearly with the length of the training sequence $Q$ [14]. The number of operations required to perform one iteration of the algorithm is a measure of computational complexity. Operations consist of multiplications, divisions, and additions. Table I compares the computational complexities of four important adaptive algorithms: LMS, RLS, FTF, and LSL. All those operations use complex operands and $Q$ is the length of the training sequence. It is worth mentioning that the numerically stable QRD-RLS algorithm also achieves $O(Q)$ complexity [14].

From Table I, the following observations can be made. The LMS algorithm is the least demanding in computational complexity. But the LMS algorithm has a slow convergence rate. The other algorithms belong to the recursive least squares family with much faster convergence rates. For a software radio smart antenna with $L$ channels and $K$ parallel beamformers for each channel, the overall complexity of the software beamformer is $K L$ times that of the Table I entries. Another way to update the weight vectors is to employ the blind methods which do not need training sequences. But in general, the complexity for a single channel is more than $O\left(Q^{2}\right)$.

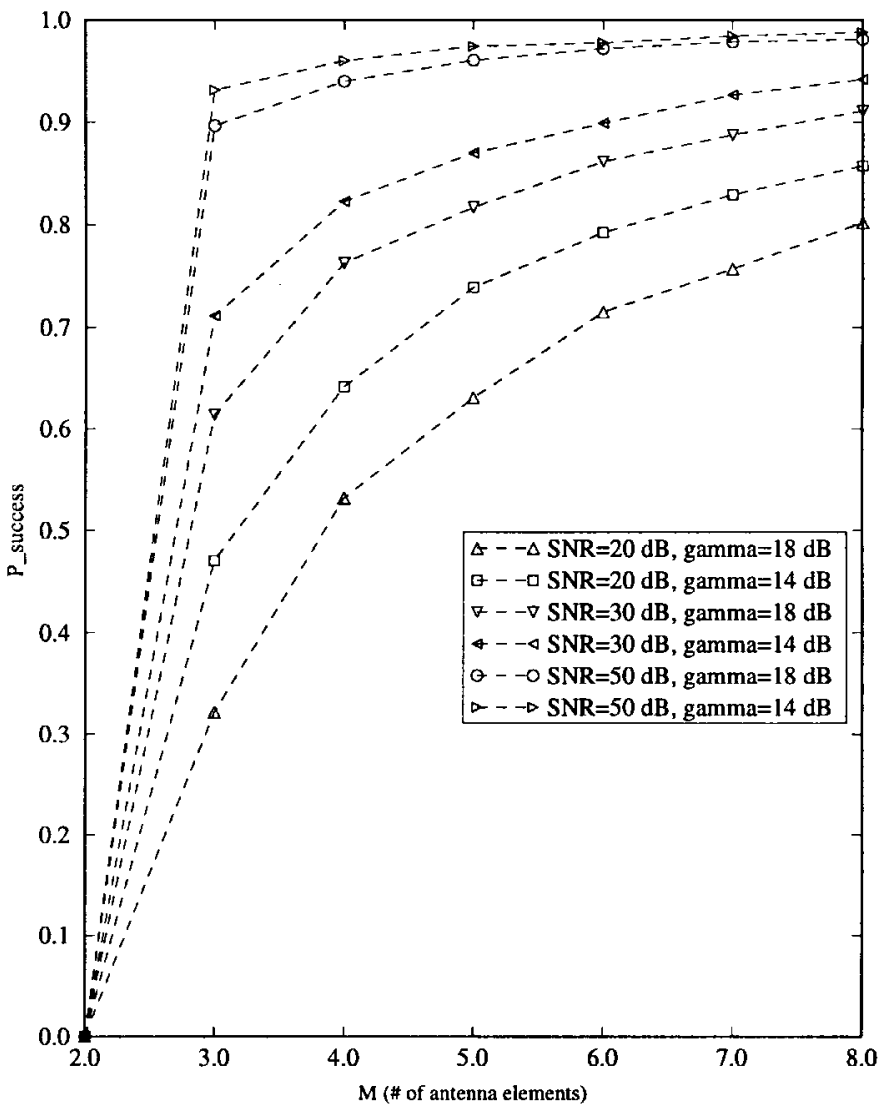

Fig. 13. Success probability $p_{3 \mid 2, M}$ for a 3-beam adaptive array for different values of $M$ (\# of antenna elements), SNR and threshold $\gamma$.

\section{APPLICATIONS}

This section presents two applications of smart antennas in a wireless network. In the first example, the smart antenna is deployed to reduce the blocking probability (or forced termination) of the handoff calls. The second example illustrates the use of a smart antenna in a CDMA network to achieve a more efficient power control algorithm. These two applications are computationally demanding, but can be implemented in software efficiently.

\section{A. Traffic and Handoff Improvement with Smart Antennas}

This section analyzes the network with adaptive array under traffic policies proposed in [12] and [13]. In this network model [14] it is assumed that when a new call (handoff or originating call) arrives, the adaptive array points one beam toward that user, and assigns one channel out of those $L$ channels to that user. Each channel $c_{i}$ for $i=1,2, \cdots, L$ may be assigned to one of the $K$ users by $K$ separate beams, using the $K$ beamformers in parallel for each channel, as shown in Fig. 10. If the first beam of all channels is occupied, the new call is assigned to another beam. If there are no multipaths in the system, with $K$ beamformers for each channel and $L \mathrm{RF}$ frequency channels, at most $K L$ users can be accepted into the system. On the other hand, if there are multipath signals with effective $\alpha$ paths per user, then the number of antennas has to increase to null those multipaths. In this case, the effective number of usable channels would be $K L / \alpha$. The flowchart 


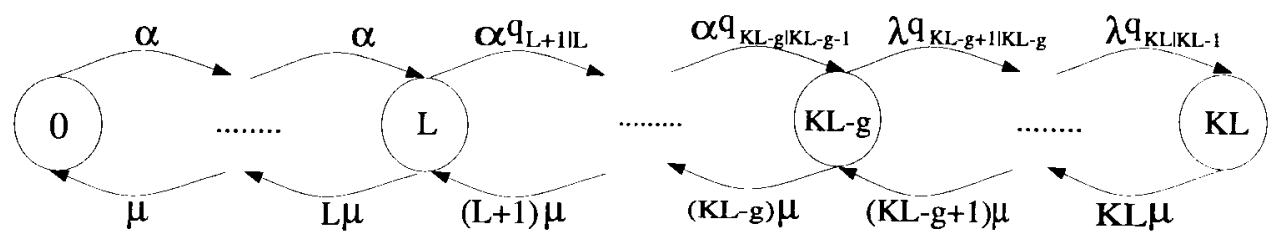

Fig. 14. State transition diagram of discrete Markov chain, the network model of the system with an adaptive array at the base station and guard channels.

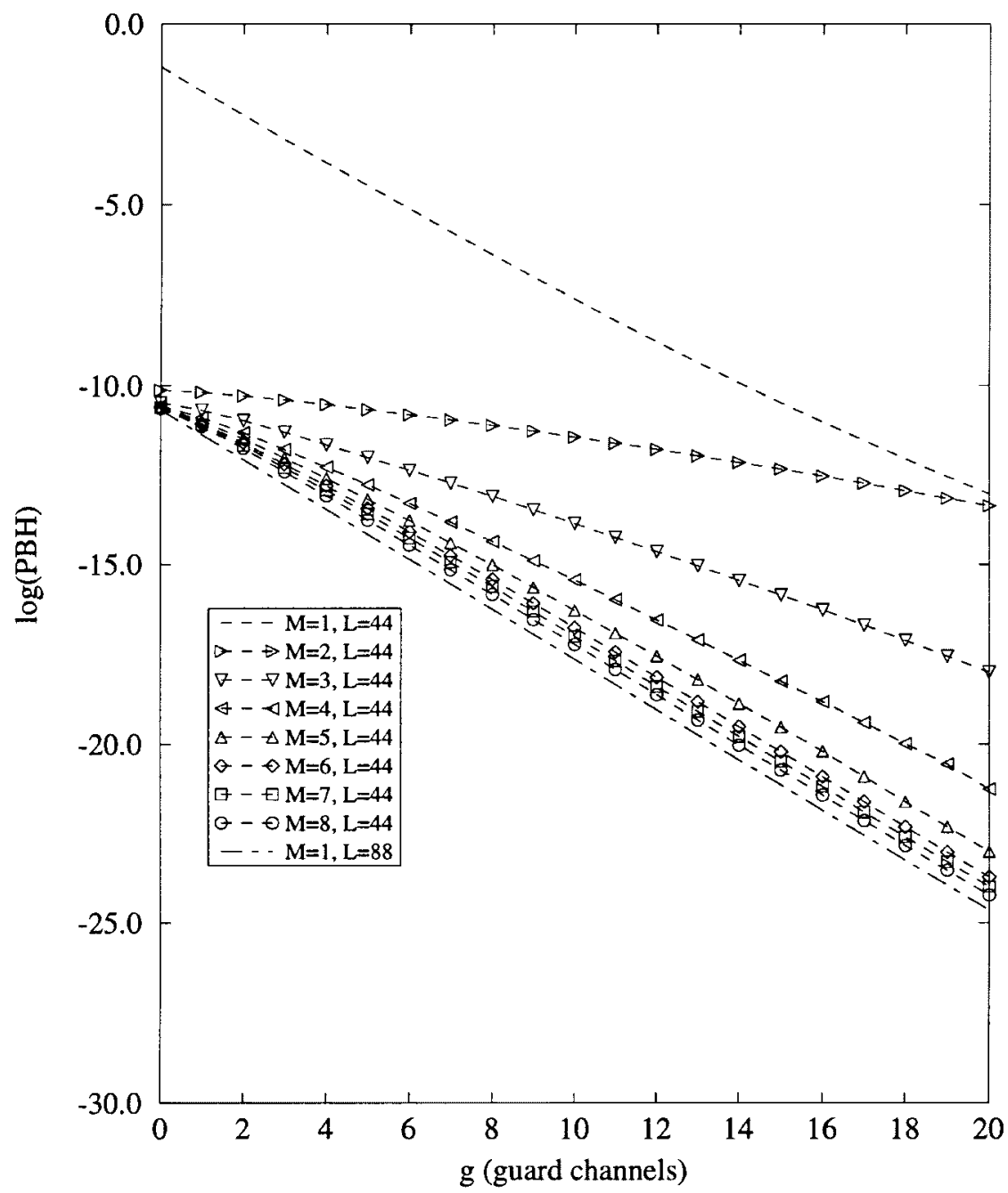

Fig. 15. Blocking probabilities of hand off calls $\left(B_{H}\right)$ for different numbers of antenna elements $M$, with SNR $=20, \gamma=18 \mathrm{~dB}, a=40, c=8 \beta=4$, and a two-beam adaptive array systsem.

of the channel assignment with smart antennas is illustrated in Fig. 11.

Assume that $(i-1)$ cochannel transmitters successfully share the same channel. For acceptable link quality, the newly arrived $i$ th transmitter shares that channel if $\Gamma_{i} \geq \gamma$, where $\Gamma_{i}$ is given by (10) and $\gamma$ is a system parameter which is dictated by the governing standard. For instance, in the IS-54 standard, $\gamma$ is $14 \mathrm{~dB}$, while in AMPS, it is $18 \mathrm{~dB}$. The probability of establishing an acceptable link, i.e., $P\left(\Gamma_{i}>\gamma\right)$, is estimated by Monte Carlo simulation. In the network model, the probability density function for the angular position of the transmitters in the system is assumed to be $f_{\Theta_{j}}\left(\theta_{j}\right)$ for $1 \leq j \leq J$. The Monte Carlo simulations of the success probabilities $p_{2 \mid 1, M}$ and $p_{3 \mid 2, M}$, for two-beam and three-beam adaptive arrays, respectively (with $M$ antenna elements), are shown in Figs. 12 and 13. A call admission control (CAC) is required to avoid degradations in system performance. If $\Gamma_{i}<\gamma$ or $i>K$, that user is not accepted into the system. The probability of the event $\Gamma_{i} \geq \gamma$ in the system, would yield the success probability $p_{i \mid i-1, M}$ that the $i$ th transmitter can share the same channel, given that $i$ transmitters are already using that channel. Clearly, the success probability $p_{i \mid i-1, M}$ depends on $M$. Since there are $L$ distinct channels in the system and each channel may be reused up to $K$ times, define the probability of successful reception of the $(n+1)$ th user into the system given that there are already $n$ users in the system as

$$
q_{N_{t+1} \mid N_{t}}\left(N_{t+1}=n+1 \mid N_{t}=n\right)
$$

where $N_{t}$ is the number of users in the system at time $t$ before a new call (user) arrives into the system and $t$ is the time 


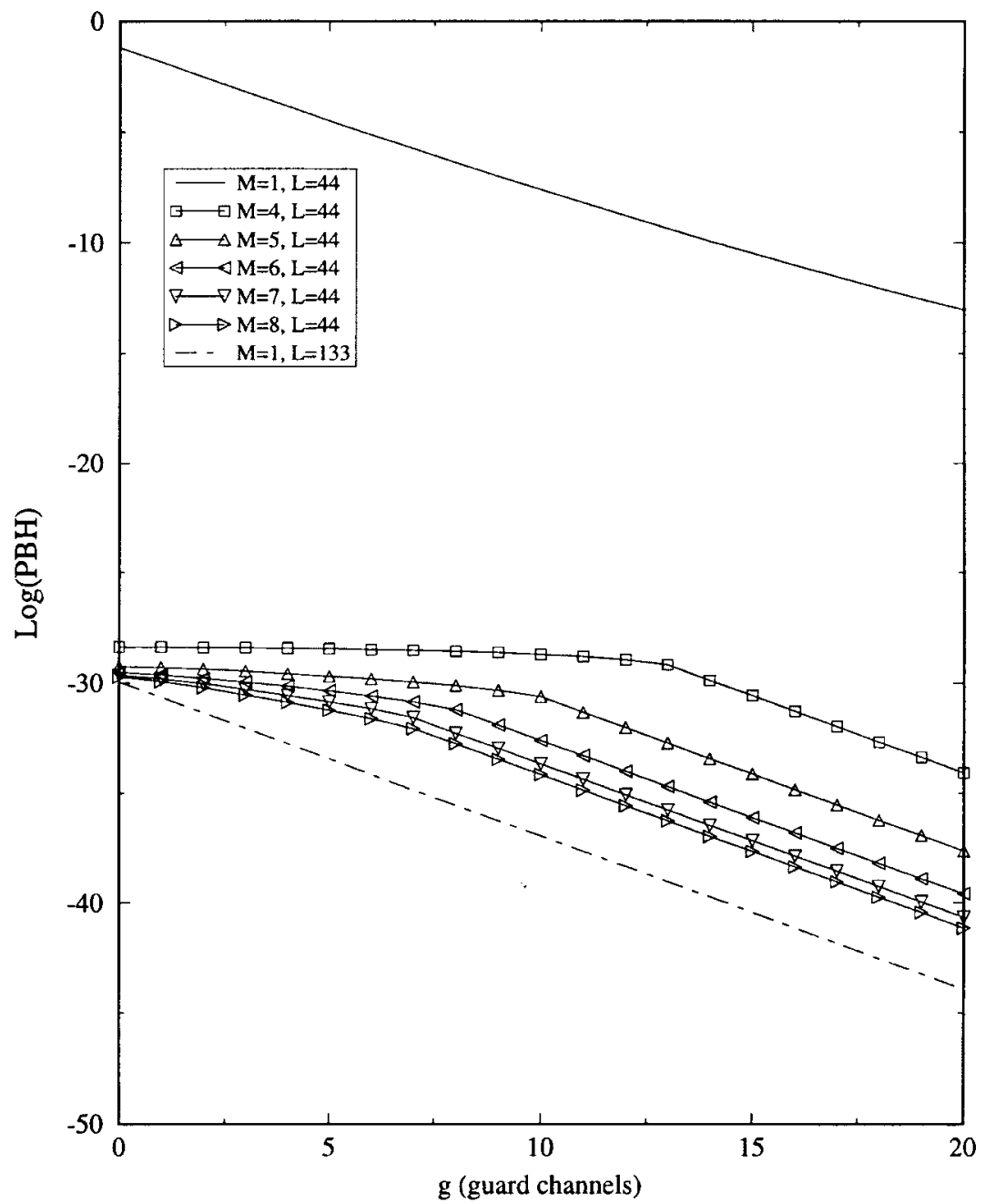

Fig. 16. Blocking probabilities of hand off calls $\left(B_{H}\right)$ for different numbers of antenna elements $M$, with SNR $=20, \gamma=18 \mathrm{~dB}, a=40, c=8 \beta=4$, and a three-beam adaptive array system.

index which increases by 1 at each epoch corresponding to a new handoff or originating call. For a $K$-beam adaptive array system, the success probabilities $q_{n+1 \mid n}$ are [14]

$$
\begin{aligned}
q_{n+1 \mid n}= & \sum_{\left\{x_{2} \cdots x_{K} \mid x_{K} \leq \cdots \leq x_{2}\right\}} \hat{q}\left(n+1, x_{2}, \cdots, x_{K}\right) \\
& \cdot p\left[\left(x_{2}, \cdots, x_{K}\right) \mid x_{2}+\cdots+x_{K}=n-L\right]
\end{aligned}
$$

where $\hat{q}\left(n+1, x_{2}, \cdots, x_{K}\right)=1-\left(1-p_{2 \mid 1, M}\right)^{\left(L-x_{2}\right)} \times$ $\left(1-p_{3 \mid 2, M}\right)^{\left(x_{2}-x_{3}\right)} \times \cdots \times\left(1-p_{K \mid K-1, M}\right)^{\left(x_{K-1}-x_{K}\right)}$, and $\left(x_{2}, x_{3}, \cdots, x_{K}\right)$ denotes the vector representing the number of calls assigned to the channels in the second beam up to the $K$ th beam. Let $\alpha=\lambda+\gamma, \mu=\eta+\nu, a=\alpha / \mu, b=\gamma / \mu, c=$ $\lambda / \mu$, where $\gamma$ and $\eta$ are the arrival rate and service rate for originating calls, and $\lambda$ and $\nu$ are the arrival rate and service rate for handoff calls, respectively. In the network with an adaptive array, the effective arrival rate into the cell at state $n, \lambda_{\text {eff }}(n)$ is [14], [52]

$$
\lambda_{\text {eff }}(n)= \begin{cases}\alpha q_{n+1 \mid n}, & n=0,1, \cdots, K L-g-1 \\ \lambda q_{n+1 \mid n}, & n=K L-g, \cdots, K L-1 .\end{cases}
$$

Similarly, the effective service rate in the system at a given state $n, \mu(n)=n \mu n=1,2, \cdots, K L$. Fig. 14 illustrates the queueing model of the system with adaptive arrays under a guard channel traffic policy.

Using this model, the state probabilities $P(n), n=$ $0,1, \cdots, K L$ (where $n$ is the number of ongoing calls in the cell) are derived [50], [51], and from there, the blocking probability $B_{O}$ of originating calls and the blocking probability $B_{H}$ of handoff calls are given by [14]

$$
\begin{aligned}
B_{O}= & {\left[\sum_{n=L}^{K L-g-1}\left(1-q_{n+1 \mid n}\right) \frac{a^{n}}{n !} \prod_{j=0}^{n-1} q_{j+1 \mid j}\right.} \\
& \left.+a^{K L-g} \sum_{n=K L-g}^{K L} \frac{c^{n-(K L-g)}}{n !} \prod_{j=0}^{n-1} q_{j+1 \mid j}\right] P(0) \\
B_{H}= & {\left[\sum_{n=L}^{K L-g-1}\left(1-q_{n+1 \mid n}\right) \frac{a^{n}}{n !} \prod_{j=0}^{n-1} q_{j+1 \mid j}\right.} \\
& +\sum_{n=K L-g}^{K L-1}\left(1-q_{n+1 \mid n}\right) \frac{a^{K L-g} c^{n-(K L-g)}}{n !} \prod_{j=0}^{n-1} q_{j+1 \mid j} \\
& \left.+\frac{a^{K L-g} c^{g}}{(K L) !} \prod_{j=0}^{K L-1} q_{j+1 \mid j}\right] P(0)
\end{aligned}
$$




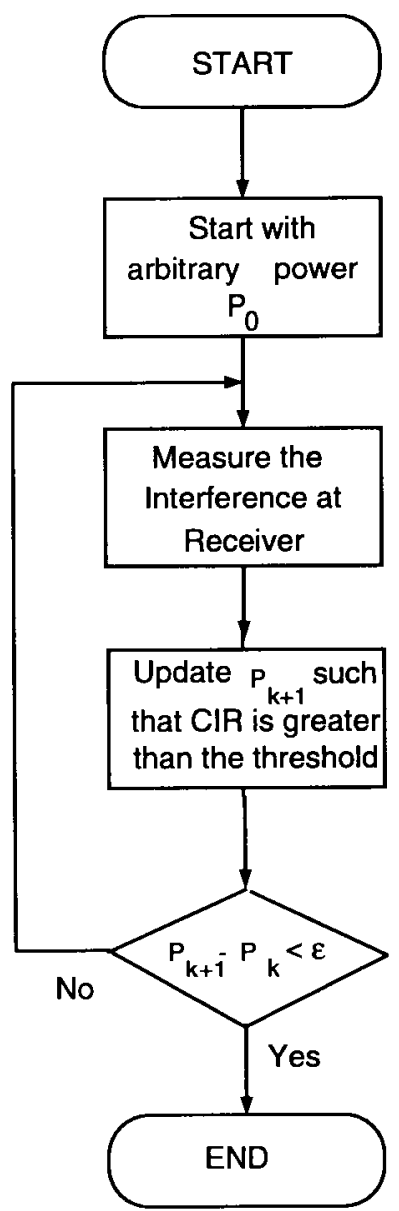

Fig. 17. Power control algorithm.

The performance of the system is evaluated with parameters drawn from [12]. Choose a cell with $L=44$ channels, with a total offered traffic of $a=40$ Erlangs (heavy traffic) and a handoff traffic of $c=8$ Erlangs. Blocking probabilities of handoff and originating calls for different values of SNR, threshold $\gamma$, and number of antenna elements $M$ are plotted in Figs. 15 and 16. In these simulations, the path loss exponent $\beta$ is assumed to be four.

Using (22), the blocking probabilities for handoff calls $B_{H}$, for different numbers of antenna elements in a two-beam and three-beam adaptive array system, are plotted in Figs. 15 and 16. These two figures illustrate that for a given SNR and threshold $\gamma$, as the number of antenna elements $M$ increases, the $B_{H}$ decrease. Because of array limitations, the blocking probability $B_{H}$ in a two-beam system $(K=2, L=44)$ is slightly higher than the $B_{H}$ for the system with $K=1, L=$ 88. The same property is observed for the three-beam system compared to the system with $K=1, L=3 \times 44$. These results confirm that a system using a two-beam adaptive array has almost the same effect as doubling the number of $\mathrm{RF}$ channels in the system. A three-beam array nearly triples the capacity [14].

\section{B. Power Control and Beamforming in CDMA Networks}

Power control is necessary to reduce the near-far effect in CDMA [53], to balance the interference in a wireless network,

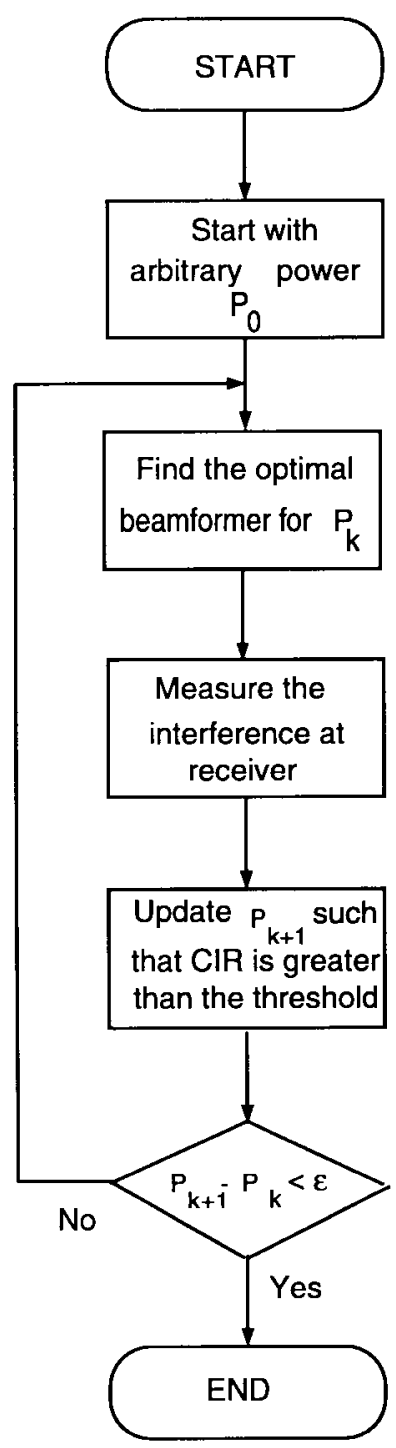

Fig. 18. The joint power control and beamforming algorithm.

and to minimize the transmitted power while the SNR is set to a threshold [54]. Distributed algorithms have been proposed [55] that adjust the power of each transmitter independently. It has been shown that these algorithms converge to the optimal power allocation that minimizes the transmitted power for each mobile while the link quality is guaranteed for each receiver. The $n$th step of the algorithm is given by

$$
P_{i}^{n+1}=\frac{\gamma}{G_{i i}}\left(\sum_{j \neq i} G_{j i} P_{j}^{n}+N_{i}\right)=\frac{\gamma}{G_{i i}} I_{i}^{n}
$$

where $P_{i}^{n}$ is the transmitted power at the $n$th iteration, and $G_{i j}$ is the link gain between the $i$ th mobile and the $j$ th base station. $N_{i}$ is the thermal noise, and $I_{i}^{n}$ the interference at the $i$ th receiver. The right-hand side of this equation is a function of the interference at the $i$ th receiver. In order to update the power, the interference $I_{i}$ is measured at each receiver, and the power is updated by multiplying a constant $\gamma / G_{i i}$. The flowchart of the algorithm is shown in Fig. 17.

Each receiver measures the interference power by calculating the average power at the output of the receiver during a 


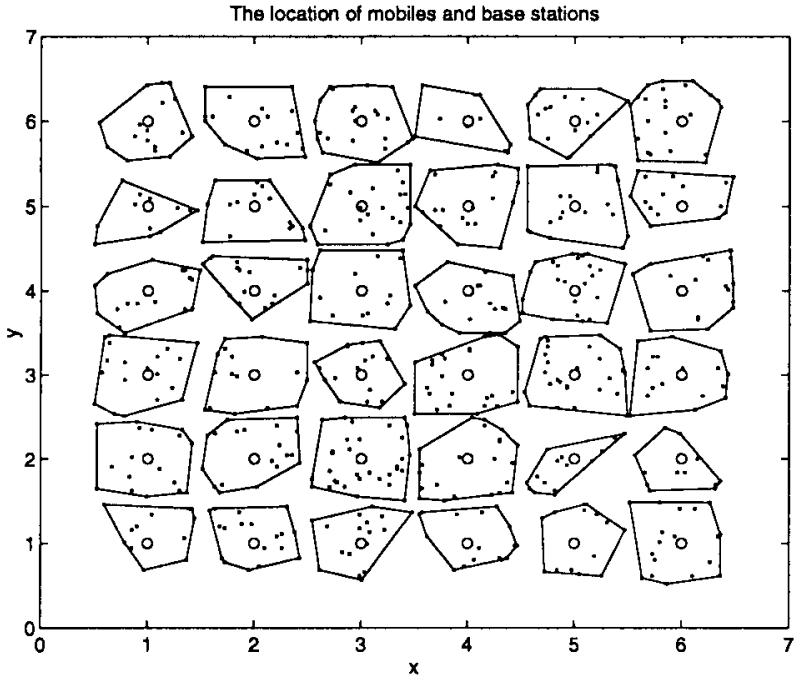

(a)

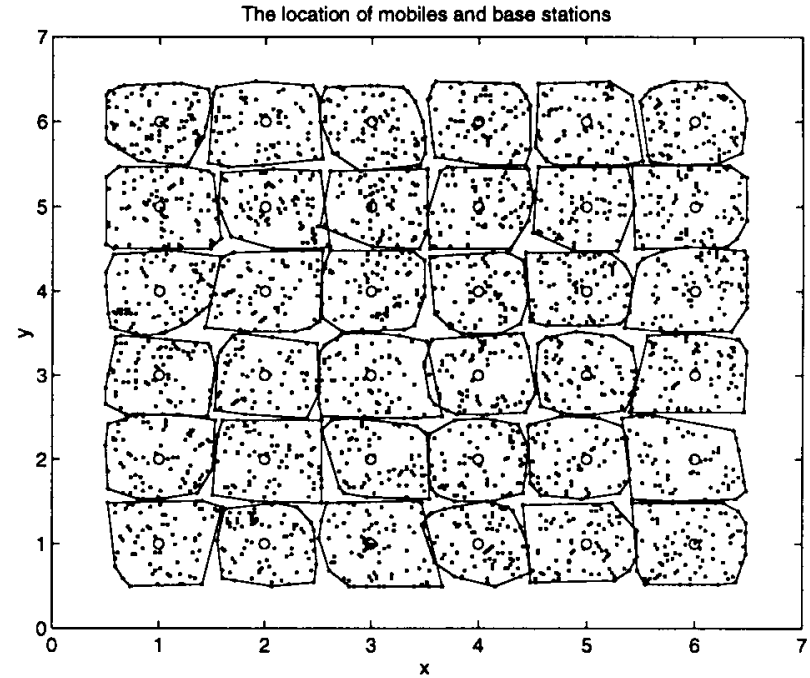

(b)

Fig. 19. Mobile and base stations locations for maximum number of users in: (a) power controlled network with single antennas and (b) power controlled network with smart antenna with four elements.

window of length $W$

$$
E_{i}=\sum_{i=1}^{W} x_{i}^{2}
$$

It subtracts the received power due to the desired transmitter power from the total received power

$$
I_{i}^{n}=E_{i}-G_{i i} P_{i}
$$

The complexity of power estimation is $4 W+1$ multiplications and $W+1$ additions. The transmitted power can be readily updated up to a few hundred times per second [53].

Joint power control and beamforming algorithms are proposed in [56]-[58]. In these algorithms, the beamforming weight vectors and power allocations are updated jointly by a distributed algorithm. The $n$th iteration of the algorithm is as follows [58].

- The minimum error is calculated by

$$
E_{i}=\sum_{k}\left|d(k)-w_{i}^{H} \boldsymbol{x}_{i}(k)\right|^{2} .
$$

- The power is updated by

$$
P_{i}^{n+1}=P_{i}^{n} \frac{\gamma}{\Gamma_{i}}=\gamma P_{i}^{n} \frac{E_{i}}{1-E_{i}} .
$$

The weight vectors are updated by the beamforming algorithm only during the training phase, and are constant between the training intervals. The complexity of the beamforming algorithms is as discussed in the previous sections. The complexity of the first step of the power control algorithm is $4 M+1$ multiplications and one addition for the calculation of $E_{\min }$ and one division and addition for updating the power. This algorithm is depicted in Fig. 18.

The above algorithm converges to the jointly optimal beamforming weight vector and power allocation such that the transmitted power is minimized [56]. In order to show the performance of the algorithm, we have simulated a network

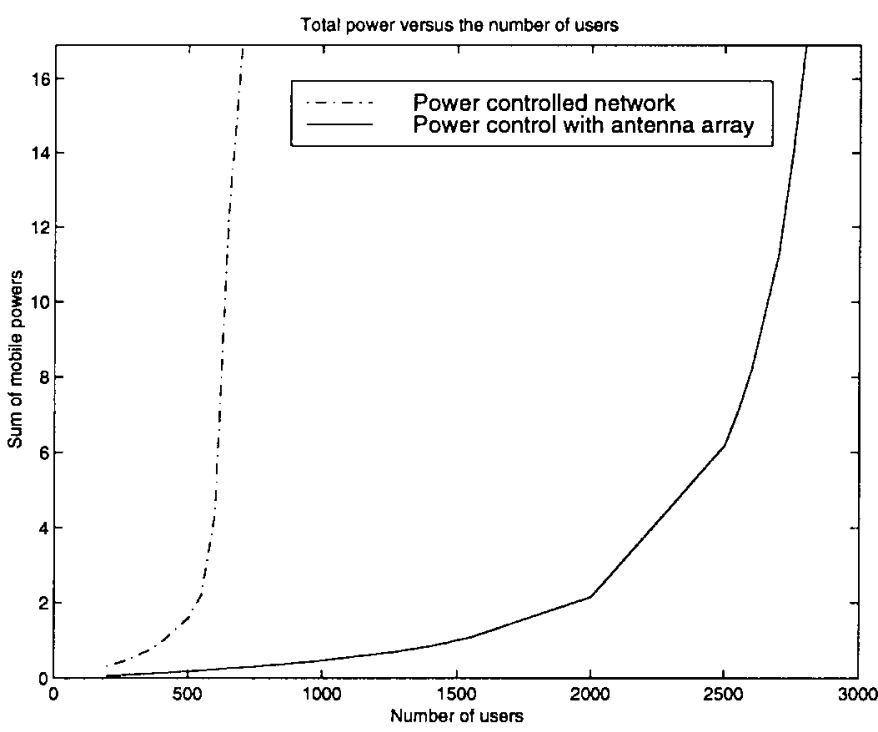

Fig. 20. The total mobile powers versus the number of users.

of mobiles and base stations where mobiles are randomly distributed in the network as illustrated in Fig. 19. The total transmitted power as a function of total number of users in the network is shown in Fig. 20. As the number of users approaches the capacity of the network, the total power is increased significantly. As illustrated in this figure, with smart antennas with four elements at the base station, for the same number of users, one can reduce the transmitted power. It is also possible to increase the maximum number of users in the network while the SNR is above a threshold.

\section{CONCLUSIONS}

This paper has presented the software radio architecture with smart antennas. The advantages of software radio implementation of wireless communication systems include flexibility for adaptive beamforming with novel algorithms. This paper also 
has reviewed the commonly employed adaptive algorithms and their complexities. Finally, two examples illustrated the effectiveness of software radio smart antenna systems in increasing the system capacity and enhancing the CDMA power control.

\section{ACKNOWLEDGMENT}

The authors wish to express their gratitude to the anonymous reviewers for their valuable suggestions and helpful comments in the preparation of this paper.

\section{REFERENCES}

[1] J. Mitola, "The software radio architecture," IEEE Commun. Mag., pp. 26-38, May 1995.

[2] __ "Software radio technology challenges and opportunities," presented at the 1st European Workshop on Software Radios, May 1997.

[3] W. Tuttlebee, "The impact of software radio," presented at the Software Radio Workshop, Brussels, Belgium, May 1997.

[4] D. L. Tennenhouse and V. G. Bose, "The spectrumWare approach to wireless signal processing," Wireless Networks.

[5] V. Bose, M. Ismert, M. Welborn, and J. Guttag, "Virtual radios," this issue, pp. 591-602.

[6] V. G. Bose, A. B. Shah, and M. Ismert, "Software radios for wireless networking," presented at IEEE Infocom'98, San Francisco, CA, Apr. 1998.

[7] T. Turletti and D. Tennenhouse, "Estimating the computational of software GSM base station," in Proc. IEEE ICC'97, Montreal, Canada, June 1997.

[8] J. Kennedy and M. C. Sullivan, "Direction finding and smart antennas using software radio architectures," IEEE Commun. Mag., pp. 62-68, May 1995.

[9] D. Everitt, "Traffic engineering of the radio interface for cellular mobile networks," Proc. IEEE (Special issue on Wireless Networks for Mobile and Personal Communications), pp. 1371-1382, Sept. 1994.

[10] , "Combined analysis of transmission and traffic in micro cellular mobile communications system," in Proc. IEEE VTC, May 1993, pp. $577-580$.

[11] B. Jabbari, G. Colombo, A. Nakajima, and J. Kulkarni, "Network issues for wireless communications," IEEE Commun. Mag., pp. 88-102, Jan. 1995.

[12] E. Posner and R. Guerin, "Traffic policies in cellular radio that minimizes blocking of handoff calls," in Proc. 11th Teletraffic Cong. (ITC 11), Kyoto, Japan, Sept. 1985, pp. 294-298.

[13] R. Guerin, "Queueing-blocking system with two arrivals stream and guard channels," IEEE Trans. Commun., vol 36, pp. 153-163, Feb. 1988

[14] J. Razavilar, "Signal processing and performance analysis for optimal resource allocation in wireless networks," Ph.D. dissertation, Elect. Eng. Dept., University of Maryland, College Park, December, 1998.

[15] S. S. Haykin, Adaptive Filter Theory. Englewood Cliffs, NJ: Prentice-Hall, 1991.

[16] A. Naguib, A. Paulraj, and T. Kailath, "Capacity improvement with base-station antenna arrays in cellular CDMA," IEEE Trans. Veh. Technol., vol. 43, Aug. 1994.

[17] B. Suard, A. Naguib, G. Xu, and A. Paulraj, "Performance analysis of CDMA mobile communication systems using antenna array," in Proc. ICASP'93, vol. IV, Apr. 1993.

[18] G. Xu, Y. Cho, A. Paulraj, and T. Kailath, "Maximum likelihood detection of co-channel communication signals via exploitation of spatial diversity," in Proc. 26th Asilomar Conf. Signals, Syst., Comput., CA, Oct. 1992.

[19] A. Klouche-Djedid and M. Fujita, "Adaptive array processing applications for mobile telephone communication," IEEE Trans. Veh. Technol., pp. 405-416, Aug. 1996

[20] S. Swales, M. Beach, D. Edwards, and J. McGeehan, "The performance enhancement of multibeam adaptive base-station antenna for cellular land mobile radio systems," IEEE Trans. Veh. Technol., pp. 56-67, Feb. 1990.

[21] T. Gebauer and H. Gockler, "Channel-individual adaptive beamforming for mobile satellite communications," IEEE J. Select. Areas Commun., pp. 439-448, Feb. 1995.

[22] S. J. Orfanidis, Optimal Signal Processing: An Introduction. New York: Macmillan, 1985.
[23] J. H. Winters, "Smart antennas for wireless systems," IEEE Personal Commun. Mag., vol. 5, pp. 23-27, Feb. 1998.

[24] B. Widrow and S. Stearns, Adaptive Signal Processing. Englewood Cliffs, NJ: Prentice-Hall, 1985.

[25] D. Donoho, "On minimum entropy deconvolution," in Applied Time Series Analysis II. New York: Academic, 1981, pp. 565-608.

[26] G. B. Giannakis and J. M. Mendel, "Identification of nonminimum phase systems using via higher order statistics," IEEE Trans. Acoust., Speech, Signal Processing, vol. 37, pp. 360-377, 1989.

[27] C. L. Nikias, "ARMA bispectrum approach to nonminimum phase system identification," IEEE Trans. Acoust., Speech, Signal Processing, vol. 36, pp. 513-525, Apr. 1988.

[28] J. K. Tugnait, "Identification of linear stochastic systems via second and fourth-order cumulant matching," IEEE Trans. Inform. Theory, vol. IT-33, pp. 393-407, May 1987.

[29] A. Benveniste, M. Goursat, and G. Ruget, "Robust identification of a nonminimum phase system: Blind adjustment of a linear equalizer in data communications," IEEE Trans. Automat. Contr., vol. AC-25, pp. 385-399, June 1980.

[30] Y. Chen and C. L. Nikias, "Blind equalization with criterion with memory nonlinearity," Opt. Eng., vol. 31, pp. 1200-1210, June 1992

[31] D. N. Godard, "Self-recovering equalization and carrier tracking in twodimensional data communication systems," IEEE Trans. Commun., vol. COM-28, pp. 1867-1875, 1980.

[32] F. Gustafsson and B. Wahlberg, "Blind equalization by direct examination of the input sequence," IEEE Trans. Commun., vol. 43, pp. 2213-2222, July 1995.

[33] G. Picchi and G. Prati, "Blind equalization and carrier recovery using a 'stop-and-go' decision-directed algorithm," IEEE Trans. Commun., vol. COM-35, pp. 877-887, 1987.

[34] Y. Sato, "A method of self-recovering equalization for multi-level amplitude modulation," IEEE Trans. Commun., vol. COM-23, pp. 679-682, June 1975 .

[35] O. Shalvi and E. Weinstein, "New criteria for blind deconvolution of nonminimum phase systems (channels)," IEEE Trans. Inform. Theory, vol. 36, pp. 312-321, Mar. 1990

[36] J. R. Treichler and B. G. Agee, "A new approach to multipath correction of constant modulus signals," IEEE Trans. Acoust., Speech, Signal Processing, vol. ASSP-31, pp. 349-372, 1983.

[37] J. R. Treichler and M. G. Larimore,"New processing techniques based on the constant modulus adaptive algorithm," IEEE Trans. Acoust., Speech, Signal Processing, vol. ASSP-33, pp. 420-431, Apr. 1985.

[38] S. Verdu, B. D. O. Anderson, and R. A. Kennedy, "Blind equalization without gain identification," IEEE Trans. Inform. Theory, vol. 39, pp. 292-297, Jan. 1993.

[39] J. K. Tugnait, "On fractionally-spaced blind adaptive equalization under symbol timing offsets using Godard and related equalizers," in Proc. IEEE ICASSP'95, vol. 3, May 1995, pp. 1976-1979.

[40] B. R. Petersen and D. D. Falconer, "Suppression of adjacent-channel, co-channel, and intersymbol interference by equalizers and linear combiners," IEEE Trans. Commun., vol. 42, pp. 3109-3118, Dec. 1994.

[41] J. Yang and S. Roy, "On joint transmitter and receiver optimization for multiple-input-multiple output (MIMO) transmission systems," IEEE Trans. Commun., vol. 42, pp. 3221-3231, Dec. 1994.

[42] J. R. Treichler and M. G. Larimore, "The tone capture properties of CMA-based interference suppressors," IEEE Trans. Acoust., Speech, Signal Processing, vol. ASSP-33, pp. 946-958, Aug. 1985.

[43] S. Mayrargue, "A blind spatio-temporal equalizer for a radio-mobile channel using the constant modulus algorithm (CMA)," in Proc. IEEE ICASSP'94, Adelaide, Australia, Apr. 1994, pp. III 317-320.

[44] D. Slock, "Blind joint equalization of multiple synchronous mobile users using oversampling and/or multiple antennas," in Proc. 28th Asilomar Conf. Signal Syst. Comput., Pacific Grove, CA, Oct. 1994, pp. $1154-1158$

[45] E. Weinstein, A. Swami, G. Giannakis, and S. Shamsunder, "Multichannel ARMA processes," IEEE Trans. Signal Processing, vol. 42, pp. 898-913, Apr. 1994.

[46] E. Weinstein, A. V. Oppenheim, M. Feder, and J. R. Buck, "Iterative and sequential algorithms for multisensor signal enhancement," IEEE Trans. Signal Processing, vol. 42, pp. 846-859, Apr. 1994.

[47] D. Yellin and E. Weinstein, "Criteria for multichannel signal separation," IEEE Trans. Signal Processing, vol. 42, pp. 2158-2168, Aug. 1994.

[48] E. Moulines, P. Duhamel, J. Cardoso, and S. Mayrargue, "Subspace methods for the blind identification of multichannel FIR filters," IEEE Trans. Signal Processing, vol. 43, pp. 516-525, Feb. 1995.

[49] A. Naguib and A. Paulraj, "Performance of CDMA cellular networks with base-station antenna arrays," in Proc. Int. Zurich Seminar Digital Commun., Zurich, Switzerland, Mar. 1994, pp. 87-100.

[50] R. B. Cooper, Introduction to Queueing Theory, 2nd ed., 1981 
[51] L. Kleinrock, Queueing Systems, Vol. I, 1985.

[52] R. A. Monzingo and T. W. Miller, Introduction to Adaptive Arrays. New York: Wiley, 1980.

[53] J. Razavilar, F. Rashid-Farrokhi, and K. J. R. Liu, "Blocking probability of handoff calls and carried traffic in wireless networks with antenna arrays," in Proc. 31st Asilomar Conf. Signals, Syst., Comput., Pacific Grove, CA, Nov. 1997, pp. 635-639.

[54] "An overview of the application of code division multiple access (CDMA) to digital cellular systems and personal cellular networks," Qualcomm Inc., May 1992.

[55] J. Zander, "Performance of optimum transmitter power control in cellular radio systems," IEEE Trans. Veh. Technol., vol. 41, pp. 57-62, Feb. 1992.

[56] _ "Distributed cochannel interference control in cellular radio systems," IEEE Trans. Veh. Technol., vol. 41, pp. 305-311, Aug. 1992.

[57] F. Rashid-Farrokhi, L. Tassiulas, and K. J. R. Liu, "Joint optimal power control and beamforming for wireless networks with antenna arrays," in Proc. IEEE Global Commun. Conf. (GLOBECOM-96), London, England, Nov. 1996, pp. I-555-559.

[58] _ "Joint optimal power control and beamforming in wireless networks with antenna arrays," IEEE Trans. Commun., vol. 46, pp. 1313-1324, Oct. 1998.

[59] F. Rashid-Farrokhi, K. J. R. Liu, and L. Tassiulas, "Transmit and receive diversity and equalization in wireless networks with fading channels," presented at IEEE Globecome'97, Phoenix, AZ, Nov. 1997.

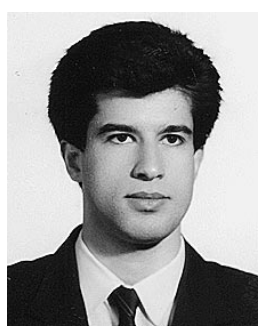

Javad Razavilar (S'89-M'99) received the B.S. degree in electrical engineering from Sharif University of Technology, Tehran, Iran, in 1989 and the M.S and $\mathrm{Ph} . \mathrm{D}$. degrees in electrical engineering from University of Maryland at College Park in 1996 and 1998, respectively.

He joined 3COM Labs, San Diego, CA, as a Senior Member of Technical Staff, in 1999. From January 1998 to September 1998 he was with Signal Processing Group (SPG) of Bay Networks Inc., Gaithersburg, MD, as a Senior DSP Software Engineer. From January 1994 to December 1998 he was a Research Assistant at institute for systems research (ISR) at University of Maryland. From January 1993 to January 1994 he was a Teaching Assistant in electrical engineering department of University of Maryland. His research interests are stochastic optimization, statistical signal processing, digital communications, information theory, networking, and wireless networks.

Dr. Razavilar is a member of Eta Kappa Nu, Gamma Xi chapter, the electrical engineering honor society. He is also a member of IEEE Information Theory, Communications, and Signal Processing Societies.

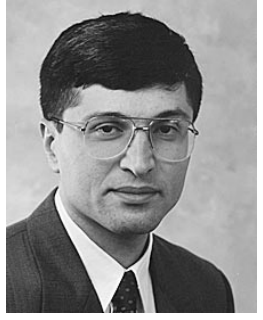

Farrokh Rashid-Farrokhi (S'88-M'97) received the B.S. and M.S. degrees (highest honors) in electrical engineering from Sharif University of Technology, Tehran, Iran, in 1988 and 1992, respectively. In 1997, he received the Ph.D. degree in electrical engineering from the University of Maryland, College Park.

He joined the Wireless Communications Research Department, Bell Laboratories, Holmdel, NJ, as a Member of Technical Staff in 1998. His research interests include array and statistical signal processing, wireless communications, and networking.

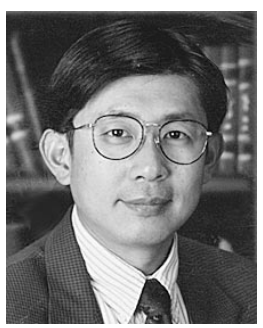

K. J. Ray Liu (S'86-M'90-SM'93) received the B.S. degree from the National Taiwan University in 1983 and the Ph.D. degree from the University of California, Los Angeles, in 1990, both in electrical engineering.

Since 1990, he has been with Electrical Engineering Department and Institute for Systems Research, University of Maryland, College Park, where he is an Associate Professor. During his sabbatical leave in 1996-1997, he was a Visiting Associate Professor at Stanford University. His research interests span various aspects of signal/image processing and communications. He has published over 160 papers, of which over 60 are in archival journals and book chapters.

Dr. Liu received numerous awards, including the 1994 National Science Foundation Young Investigator Award, the IEEE Signal Processing Society's 1993 Senior Award (Best Paper Award), the George Corcoran Award in 1994 for outstanding contributions to electrical engineering education and the 1995-1996 Outstanding Systems Engineering Faculty Award in recognition of outstanding contributions in interdisciplinary research, both from the University of Maryland, and many others. He has been an Associate Editor of IEEE Transactions on Signal Processing. He was a Guest Editor of Special Issues on Multimedia Signal Processing and Technology of Proceedings of THE IEEE, a Guest Editor of the Special Issue on Signal Processing for Wireless Communications of IEEE Journal of SeleCted AREAS in Communications, and a founding member of the Multimedia Signal Processing Technical Committee of the IEEE Signal Processing Society. He is an Editor of Journal of VLSI Signal Processing Systems, and the book series editor of the Marcel Dekker series on signal processing. He is the Coeditor of the books, High Performance VLSI Signal Processing: Volume I: System Design and Methodology; Vol. II: Algorithms, Architectures, and Applications (IEEE Press). 